\title{
Effect of Rhythmic Auditory Cueing on Aging Gait: A Systematic Review and Meta-Analysis
}

\author{
Shashank Ghai ${ }^{1, *}$, Ishan Ghai ${ }^{2}$, Alfred O. Effenberg1 \\ ${ }^{1}$ Institute for Sports Science, Leibniz University Hannover, Germany \\ ${ }^{2}$ School of Life Sciences, Jacobs University Bremen, Germany
}

[Received July 27, 2017; Revised October 28, 2017; Accepted October 31, 2017]

\begin{abstract}
Rhythmic auditory cueing has been widely used in gait rehabilitation over the past decade. The entrainment effect has been suggested to introduce neurophysiological changes, alleviate auditory-motor coupling and reduce cognitive-motor interferences. However, a consensus as to its influence over aging gait is still warranted. A systematic review and meta-analysis was carried out to analyze the effects of rhythmic auditory cueing on spatiotemporal gait parameters among healthy young and elderly participants. This systematic identification of published literature was performed according to PRISMA guidelines, from inception until May 2017, on online databases: Web of science, PEDro, EBSCO, MEDLINE, Cochrane, EMBASE, and PROQUEST. Studies were critically appraised using PEDro scale. Of 2789 records, 34 studies, involving 854 (499 young/ 355 elderly) participants met our inclusion criteria. The meta-analysis revealed enhancements in spatiotemporal parameters of gait i.e. gait velocity (Hedge's g: 0.85$)$, stride length $(0.61)$, and cadence (1.1), amongst both age groups. This review, for the first time, evaluates the effects of auditory entrainment on aging gait and discusses its implications under higher and lower information processing constraints. Clinical implications are discussed with respect to applications of auditory entrainment in rehabilitation settings.
\end{abstract}

Key words: cueing, stability, rehabilitation, cognitive-motor interference, balance, entrainment, dual task

Higher prevalence to fall with aging is a matter of concern for medical practitioners [1-3]. According to WHO, every year approximately 37 million people are seriously injured, and further 424,000 people perish from falls globally [4]. Degenerative changes in cardiovascular [5], sensorimotor (somatosensory, vestibular), and neuromuscular (cortical, extra-pyramidal, cerebellum) domains are suggested to be the main reasons often leading to falls [6-8]. Moreover, medications, depression, and anxiety are additional precipitators [9-11]. Falls impact quality of life $[12,13]$, and inflict heavy costs at both individual, economic levels [14, 15].

Studies suggest that highest incidences for falls occur during locomotion [16-18]. In fact, aging has been associated with modifications in spatiotemporal [19], electromyographic [20], and kinematic [21], gait parameters, which in-turn are important predictors for fall. For instance, clinical characteristics for reductions in gait velocity, stride length, cadence, single limb support phase, and enhancements in stride time, double limb support phase, gait variability [22, 23], have been well documented (see also Jahn, et al. [6]). The kinematic analysis also suggests a reduction in angular impulse, torque at ankle, knee, and hip joint with aging gait [24]. Together, these factors aggravate static and dynamic instability and increase predisposition to fall. Likewise, degenerative changes observed in psychological domain in elderly might also contribute in modifying stability [25,

*Correspondence should be addressed to: Dr. Shashank Ghai, Institut für Sportwissenschaft, Leibniz Universität, Am Moritzwinkel 6, Hannover 30167, Germany. E-mail address: shashank.ghai@ sportwiss.uni-hannover.de

Copyright: ( 2017 Ghai S et al. This is an open-access article distributed under the terms of the Creative Commons Attribution License, which permits unrestricted use, distribution, and reproduction in any medium, provided the original author and source are credited. 
26], and cognitive processing [27, 28]. Reelick, et al. [23], for instance, suggested a reduction in self-confidence with aging, and history of falls often leading to a peculiar "fear of falling" [29, 30]. Furthermore, this "fear" has been reported to additionally modify the stability during static, and dynamic postures [9, 31, 32]. Giladi, et al. [32], referred such modified gait pattern as a "cautious or fearful gait" [23]. Although these modifications are aimed to enhance stability during locomotion, they, in turn, develop a stiff, slow and unsteady gait pattern [33]. Moreover, this "fear of falling" or "cautious gait" might promote "internal" attentional focus [34], explicit motor control [25], and can eventually alleviate cognitive-motor interferences [35] (see also Young and Mark Williams [33]). Masters and Maxwell [27] suggested that such an attempt to consciously monitor or control an autonomic movement, such as posture, or gait might adversely affect its performance. Also, such higher information processing constraints have demonstrated detrimental effects on proprioceptive perceptions [36-38], which are integral for autonomic stability [36]. In addition, literature suggests that younger population groups, on the contrary, have a more resilient and stable psycho-physiological stature $[35,39]$. However, falls are not uncommon [10]. Possibly, environmental [10], and lifestyle factors might play a considerable role [40]. Schabrun, van den Hoorn, Moorcroft, Greenland and Hodges [41] reported texting and reading while walking (common among youngsters) to adversely impact gait stability [42], by increasing cognitive-motor interferences [43]. Consequently, such higher attentional constraints predisposing to falls might possess serious life-threatening consequences under "high-stress" environments [8, 44], for both younger and elderly age groups.

Several strategies have been suggested in literature to curb these psycho-physiological deficits, such as pharmacotherapy (Methylphenidate) [5], virtual-reality [45], biofeedback [46], physical/occupational therapy [47], physical exercise [48], dance [49], treadmill [50], external sensory cueing [51, 52], martial arts [53, 54], dual-task training [5, 36], and more [55]. Amongst these, external sensory entrainment in rehabilitation is an emerging yet under-evaluated area of interest [56]. For instance, external auditory cueing can enhance motor performance in patients with sensorimotor deficits [57], even better vis-a-vis tactile and visual entrainment [5659]. Possibly, due to lower rhythm perceptional thresholds for auditory cortex [56, 60, 61], rich neural connectivity [52,62,63], and better temporal precision [52, 62, 63]. Moreover, published literature suggests beneficial effects auditory entrainment during gait amongst patients affected from traumatic neurological injuries [64], multiple sclerosis [65], stroke [66], parkinsonism [57], and even healthy young and elderly participants [67, 68]. The auditory entrainment might supplement sensory deficits present in fall prone individuals [69], and aid in performance by mediating multifactorial neurophysiological changes [52, 70], enhancing auditory imagery [71-74], reducing variability in musculoskeletal activation [75], and possibly cognitive-motor interference $[67,76]$.

Additionally, rhythmic auditory entrainment is cheap [77], viable [78], easy to follow and has shown enhancements even during unsupervised home-based training programs $[79,80]$. This intervention can be a useful rehabilitation tool in middle and lower income countries, where poor healthcare services [81], might precipitate to majority of the fall related deaths [4]. Thereby, strongly warranting the need for such economical, and efficient rehabilitation techniques.

High-quality systematic reviews and meta-analyses have been carried out to evaluate the beneficial effects of rhythmic auditory cueing on gait in patients affected from neurological conditions, such as stroke, and parkinsonism $[57,58,66]$. However, to the best of our knowledge, no review to date has analyzed the effects of rhythmic auditory cueing on aging gait. Therefore, we attempted to develop a state of the art knowledge for the use of rhythmic auditory cueing in gait rehabilitation across healthy population groups. The main aim of this review is to understand the effects of auditory entrainment on spatiotemporal, variability parameters for gait among young, and elderly age groups. The review also discusses possible applications of auditory entrainment in rehabilitation and activities for daily living.

\section{METHODS}

This review was conducted according to the guidelines outlined in Preferred Reporting Items for Systematic Reviews and Meta-analysis: The PRISMA statement [82].

\section{Data sources and search strategy}

Academic databases such as Web of science, PEDro, EBSCO, MEDLINE, Cochrane, EMBASE and PROQUEST were searched from inception until July 2017. A sample search strategy has been provided in (Supplementary Table 1).

\section{Data extraction}

Upon selection for review, the following data were extracted from each article; author, date of publication, selection criteria, sample size, sample description (gender, age, health status), disease duration, intervention, characteristics of auditory feedback, dual-task, outcome 
measures, results, and conclusions. The data were then summarized and tabulated (Table 1).

The inclusion criteria for the studies was (i) Performed studies were either randomized controlled trials, cluster randomized controlled trials or controlled clinical trials; (ii) Studies reporting reliable and valid spatiotemporal gait parameters (iii) Studies reporting dynamic aspects of gait stability (iv) Studies qualified PEDro methodological quality scale ( $\geq 4$ score); (v) Experiments conducted on human participants; (vi) Published in a peer-reviewed academic journal; (vii) Articles published in English and German languages.

\section{Quality \& risk of bias assessment}

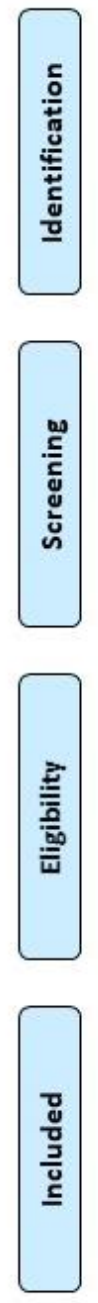

The quality of the studies was assessed using the PEDro methodological quality scale [83]. The scale consists of 11 items addressing external validity, internal validity, and interpretability and can detect potential bias with fair to good reliability [84], and validity [83]. A blinded rating of the methodological quality of the studies was carried out by the primary reviewer. Ambiguous issues were discussed with second (IG), third (AOE) reviewer and consensus was reached. Included studies were rated, and interpreted according to scoring of 9-10, 6-8 and 4-5 considered of "excellent", "good" and "fair" quality [85], respectively. Inadequate randomization, non-blinding of assessors, no intention to treat analysis and no measurement of compliance were considered as major threats to biasing [86].

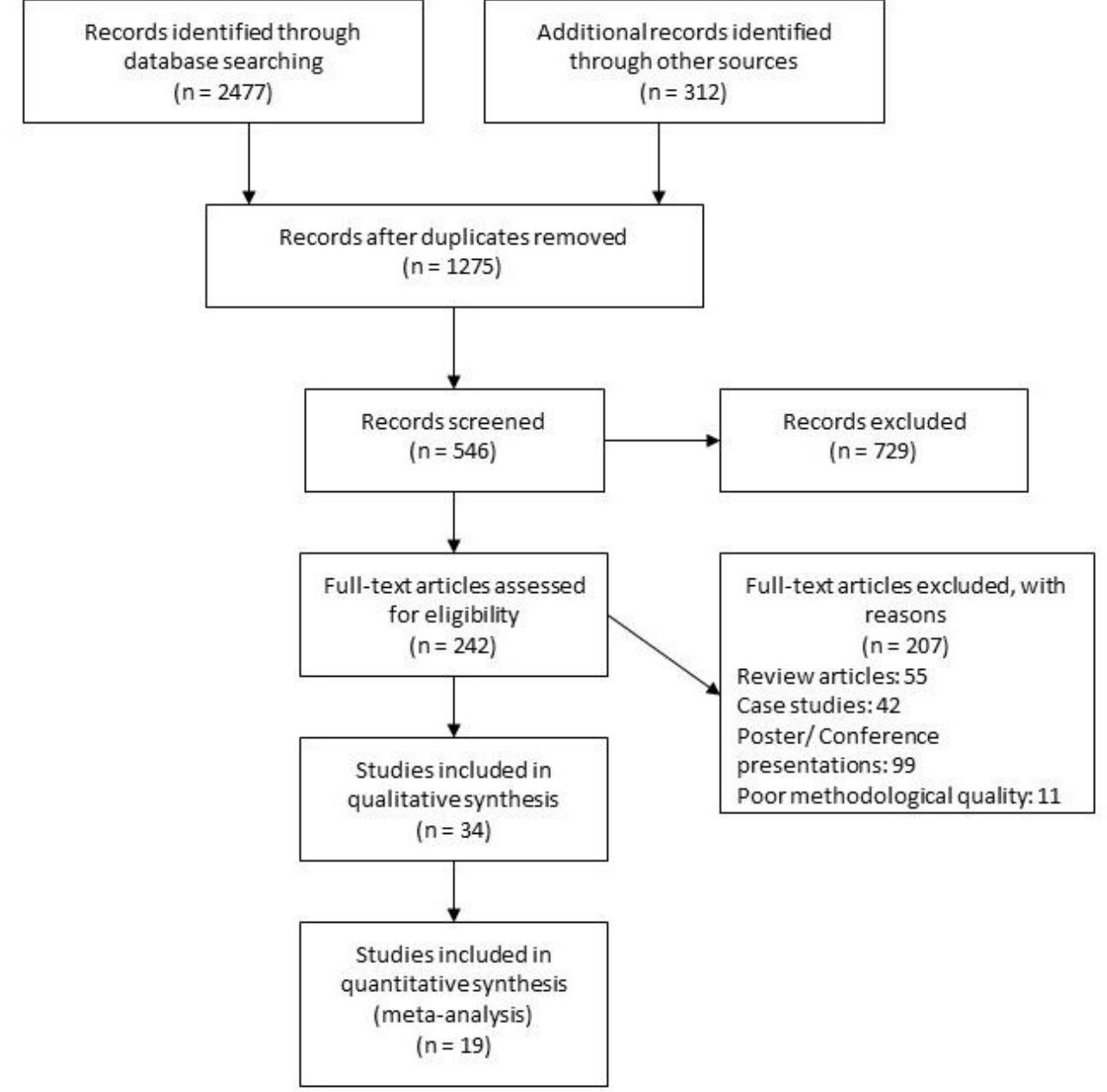

Figure 1. PRISMA flow chart for the inclusion of studies. 


\section{Data Analysis}

This systematic review included a meta-analysis approach [87]. The presence and lack of heterogeneity asserted the use of either random or fixed effect meta-analysis [88], respectively. A narrative synthesis of the findings structured around the intervention, population characteristics; methodological quality (Table 1) and the type of outcome are provided. Likewise, summaries of intervention effects for each study are also provided in a tabular form (Table 1). A meta-analysis was conducted between pooled studies using CMA (Comprehensive meta-analysis V 2.0, USA). Heterogeneity between the studies was assessed using $\mathrm{I}^{2}$ statistics. The data in this review was systematically distributed and for each available variable pooled, dichotomous data was analyzed and forest plots with $95 \%$ confidence intervals are plotted. The weighted effect sizes are reported as Hedge's g [89]. Thresholds for interpretation of effect sizes were as follows; a standard mean effect size of 0 means no change, negative effect size means a negative change, mean effect size of 0.2 considered a small effect, 0.5 a medium effect and 0.8 a large effect [90]. Interpretation of heterogeneity via $\mathrm{I}^{2}$ statistics was as; $0-0 \%, 25 \%, 75 \%$ as negligible, moderate and substantial heterogeneity, respectively. Meta-analysis reports including heterogeneity among studies were evaluated to determine the reason of heterogeneity, and the included studies were then pooled separately and analyzed again. The alpha level was set at $95 \%$.

\section{RESULTS}

\section{Characteristics of included studies}

Our initial search yielded a total of 2789 studies, which on implementing our inclusion/exclusion criteria, were reduced to thirty-four (Fig. 1). Data from the included studies have been summarized in (Table 1). Of the thirtyfour included studies, one was randomized controlled trial, and thirty-three were controlled clinical trials.

Table 1. Studies analyzing the effects of rhythmic auditory cueing on gait.

\begin{tabular}{|c|c|c|c|c|c|c|}
\hline Author & $\begin{array}{l}\text { Sample } \\
\text { description, } \\
\text { age: }(M \pm \\
\text { S.D years) }\end{array}$ & $\begin{array}{l}\text { PEDro } \\
\text { score }\end{array}$ & Assessment tools & Research design & $\begin{array}{l}\text { Auditory feedback } \\
\text { elements }\end{array}$ & Conclusion \\
\hline $\begin{array}{l}\text { Dotov, et al. } \\
{[100]}\end{array}$ & $\begin{array}{ll}7 \mathrm{~F}, & 12 \mathrm{M} \\
(60) & \end{array}$ & 6 & 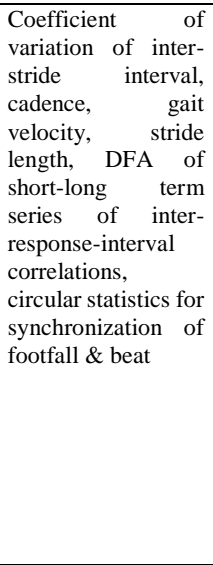 & $\begin{array}{l}\text { Pre-test, gait } \\
\text { performance } \\
\text { with/without RAC } \\
\text { (no variability, } \\
\text { biological } \\
\text { variability, non- } \\
\text { biological } \\
\text { variability; } \\
\text { randomized), post- } \\
\text { test }\end{array}$ & 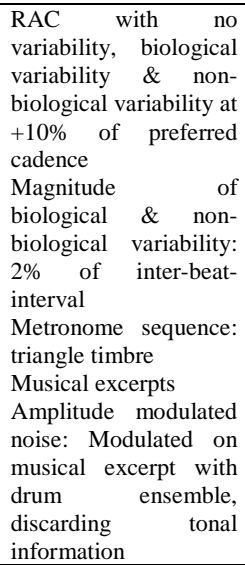 & $\begin{array}{l}\text { Significant enhancement in coefficient of } \\
\text { variation for inter-stride interval after } \\
\text { RAC in all conditions. } \\
\text { Significant effect of RAC that was } \\
\text { amplitude modulated for biological } \\
\text { variability as compared to IC on short- } \\
\text { long term correlation for term series of } \\
\text { inter-response-interval correlations. } \\
\text { Enhanced synchronization, cadence but } \\
\text { reduced short-long term correlation for } \\
\text { term series of inter-response-interval } \\
\text { correlations during metronome based IC } \\
\text { as compared to feedback with amplitude } \\
\text { modulated for biological variability. }\end{array}$ \\
\hline $\begin{array}{l}\text { Maculewicz, } \\
\text { et al. [141] }\end{array}$ & $\begin{array}{l}5 \mathrm{~F}, \quad 15 \mathrm{M} \\
(24.4 \pm 3.2)\end{array}$ & 4 & $\begin{array}{l}\text { Mean square error } \\
\text { for the asynchrony } \\
\text { between target \& } \\
\text { performed measure } \\
\& \text { trend of tempo } \\
\text { change obtained } \\
\text { from slope of line } \\
\text { fitted to measured } \\
\text { tempo, } \\
\text { questionnaire }\end{array}$ & $\begin{array}{l}\text { Gait performance } \\
\text { with/without real- } \\
\text { time auditory } \\
\text { feedback (adaptive), } \\
\text { RAC (constant) \&/or } \\
\text { haptic feedback, } \\
\text { with instructions to } \\
\text { perform gait at } \\
\text { preferred cadence or } \\
\text { the tempo of the } \\
\text { sound }\end{array}$ & 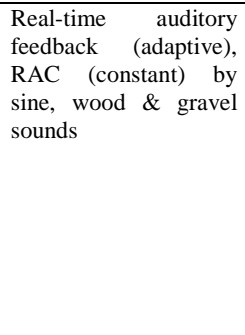 & $\begin{array}{l}\text { Significantly enhanced step wise } \\
\text { interaction with real time auditory } \\
\text { feedback with (sinusoid >wood }>\text { gravel). } \\
\text { Significant reduction in asynchrony with } \\
\text { audio-haptic feedback \& real-time } \\
\text { auditory feedback as compared to no } \\
\text { feedback. } \\
\text { Significant enhancement in comfort for } \\
\text { perceiving haptic \& audio-haptic } \\
\text { feedback as compared to haptic only or } \\
\text { no feedback in self-reported } \\
\text { questionnaire. }\end{array}$ \\
\hline $\begin{array}{l}\text { Schreiber, et } \\
\text { al. [97] }\end{array}$ & $\begin{array}{l}5 \mathrm{~F}, \quad 12 \mathrm{M} \\
(37.4 \pm 15.7)\end{array}$ & 4 & $\begin{array}{l}\text { Cadence, gait speed, } \\
\text { rhythmicity, stance } \\
\text { time, double } \\
\text { support time, gait } \\
\text { symmetry, step } \\
\text { length, stride length, }\end{array}$ & $\begin{array}{l}\text { Gait performance } \\
\text { with/without RAC } \\
\text { cueing at preferred, } \\
\text { reduced cadence } \\
\text { (instructions \& } \\
\text { cueing randomized) }\end{array}$ & $\begin{array}{l}\text { RAC at preferred \& } \\
\text { reduced cadence }\end{array}$ & $\begin{array}{l}\text { Significantly reduced gait speed with } \\
\text { RAC at preferred cadence as compared to } \\
\text { preferred speed gait without cueing. } \\
\text { No effect of RAC on cadence, } \\
\text { rhythmicity, stance time, double support } \\
\text { time, gait symmetry for RAC at preferred }\end{array}$ \\
\hline
\end{tabular}




\begin{tabular}{|c|c|c|c|c|c|c|}
\hline & & & $\begin{array}{l}\text { step width, EMG } \\
\text { activity of (tibialis } \\
\text { anterior, soleus, } \\
\text { gastrocnemius } \\
\text { medialis, vastus } \\
\text { medialis, rectus } \\
\text { femoris, } \\
\text { semitendinosus, } \\
\text { gluteus medius \& } \\
\text { gluteus maximus), } \\
\text { kinematics for } \\
\text { pelvis, hips, knees } \\
\text { \& ankle joint } \\
\text { (sagittal, frontal, } \\
\text { transverse plane) }\end{array}$ & & & $\begin{array}{l}\text { or reduced cadence as compared to no } \\
\text { cueing. } \\
\text { Significantly reduced step width with } \\
\text { RAC at reduced cadence as compared to } \\
\text { reduced speed gait without cueing. } \\
\text { Significantly enhanced step length with } \\
\text { RAC at reduced cadence as compared to } \\
\text { reduced speed gait without cueing. } \\
\text { Significant differences for ankle } \\
\text { dorsiflexion, hip flexion \& hip abduction } \\
\text { of the gait cycle with RAC at reduced } \\
\text { cadence as compared to reduced speed } \\
\text { gait without cueing. }\end{array}$ \\
\hline $\begin{array}{l}\text { Hamacher, et } \\
\text { al. }[104]\end{array}$ & $\begin{array}{l}\text { Young: } 8 \mathrm{~F} \\
12 \mathrm{M} \\
(24.9 \pm 4.1) \\
\text { Old: } 11 \mathrm{~F}, \\
9 \mathrm{M} \\
(67.4 \pm 5.3)\end{array}$ & 5 & $\begin{array}{l}\text { Stride length, } \\
\text { minimum foot } \\
\text { clearance, stride } \\
\text { time, stride to stride } \\
\text { analysis (mean \& } \\
\text { coefficient of } \\
\text { variation) }\end{array}$ & $\begin{array}{l}\text { Gait performance } \\
\text { with/without dual- } \\
\text { task (arithmetic } \\
\text { subtraction in 3's } \\
\text { task) \&/ RAC } \\
\text { (randomized) }\end{array}$ & $\begin{array}{l}\text { RAC at preferred } \\
\text { cadence }\end{array}$ & $\begin{array}{l}\text { Significant enhancement in stride length, } \\
\text { stride time with RAC (with/without dual- } \\
\text { task) in both younger \& older adults. } \\
\text { Significantly enhanced coefficient of } \\
\text { variation of stride time in older } \\
\text { participants under dual-task condition \& } \\
\text { with RAC } \\
\text { Enhancement in coefficient of variation } \\
\text { of stride to stride in older participants } \\
\text { under dual-task condition \& with RAC }\end{array}$ \\
\hline Terrier [96] & $\begin{array}{l}22 \mathrm{~F}, \quad 14 \mathrm{M} \\
(33 \pm 10)\end{array}$ & 4 & $\begin{array}{l}\text { DFA of coefficient } \\
\text { of variability for } \\
\text { stride time, stride } \\
\text { length, stride speed, } \\
\text { stride length, stride } \\
\text { speed \& stride time }\end{array}$ & $\begin{array}{l}\text { Gait performance on } \\
\text { treadmill } \\
\text { with/without visual } \\
\text { (stepping stones), } \\
\text { RAC }\end{array}$ & $\begin{array}{l}\text { RAC at preferred } \\
\text { cadence }\end{array}$ & $\begin{array}{l}\text { Significant reduction in stride time \& } \\
\text { stride speed with RAC as compared to no } \\
\text { cueing. } \\
\text { No effect on coefficient of variation for } \\
\text { stride length, stride time \& stride speed } \\
\text { (mean \& coefficient of variation) with } \\
\text { RAC }\end{array}$ \\
\hline $\begin{array}{l}\text { Roerdink, et } \\
\text { al. [162] }\end{array}$ & $\begin{array}{ll}5 \mathrm{~F}, & 7 \mathrm{M} \\
(28 \pm 6) & \end{array}$ & 5 & $\begin{array}{l}\text { Stride-to-stride } \\
\text { DFA for persistence } \\
\text { of stride time, stride } \\
\text { length, stride speed } \\
\& \text { anterior-posterior } \\
\text { center of pressure } \\
\text { sway }\end{array}$ & $\begin{array}{l}\text { Treadmill gait } \\
\text { performed } \\
\text { with/without RAC } \\
\text { with isochronous } \\
\text { metronome \& non- } \\
\text { isochronous } \\
\text { metronome } \\
\text { containing inter-beat } \\
\text { interval sequences } \\
\text { with distinct scaling } \\
\text { exponents } \\
\text { (randomized) }\end{array}$ & $\begin{array}{l}\text { RAC with (IC) } \\
\text { containing equidistant } \\
\text { inter-beat interval \& } 4 \\
\text { (non-isochronous) } \\
\text { metronome containing } \\
\text { inter-beat interval } \\
\text { sequences with distinct } \\
\text { scaling exponents } \\
\text { Frequency: } 600 \mathrm{~Hz} \\
\text { RAC with mean inter- } \\
\text { beat intervals being } \\
\text { equal to mean stride } \\
\text { time of preferred } \\
\text { cadence. }\end{array}$ & $\begin{array}{l}\text { Significant effect of IC cueing for } \\
\text { changing the stride-to-stride fluctuations } \\
\text { of stride length \& stride time to anti- } \\
\text { persistent \& vice versa for the non-IC. } \\
\text { Significant effect of isochronous \& non- } \\
\text { isochronous metronome cueing for } \\
\text { changing the stride-to-stride fluctuations } \\
\text { of stride speed to anti-persistent for both } \\
\text { the cueing. }\end{array}$ \\
\hline $\begin{array}{l}\text { Wright, } \\
\text { Spurgeon } \\
\text { and Elliott } \\
{[163]}\end{array}$ & $\begin{array}{l}8 \mathrm{~F}, 2 \mathrm{M} \mathrm{(20-} \\
33)\end{array}$ & 5 & $\begin{array}{l}\text { Mean asynchrony, } \\
\text { step time variability } \\
\text { \& mean percentage } \\
\text { step correction }\end{array}$ & $\begin{array}{l}\text { Gait performance } \\
\text { with/without RAC } \\
\text { \&/or visual cueing }\end{array}$ & $\begin{array}{l}\text { RAC, } 500 \mathrm{~ms} \mathrm{(cue} \\
\text { duration } 30 \mathrm{~ms} \text { ), } 800 \mathrm{~Hz}\end{array}$ & $\begin{array}{l}\text { Significant enhancement in \& mean } \\
\text { percentage step correction with audio \& } \\
\text { audio-visual cueing as compared to only } \\
\text { visual cueing } \\
\text { Significant reduction in mean synchrony } \\
\text { of step with RAC with audio-visual } \\
\text { cueing as compared to only audio or } \\
\text { visual cueing. } \\
\text { Significant reduction in step time } \\
\text { variability with audio \& audio-visual } \\
\text { cueing as compared to only visual cueing }\end{array}$ \\
\hline $\begin{array}{l}\text { Young, et al. } \\
{[138]}\end{array}$ & $\begin{array}{l}\text { 6F, } 4 \mathrm{M} \\
(63.9 \pm 4) \\
\text { II: same as I } \\
\text { III: same as } \\
\text { I }\end{array}$ & 5 & $\begin{array}{l}\text { I: Mean step length, } \\
\% \text { change stride } \\
\text { length, mean step } \\
\text { duration, \% change } \\
\text { in variability of } \\
\text { stride length, } \\
\text { duration } \\
\text { II: same as I } \\
\text { III: same as I }\end{array}$ & $\begin{array}{l}\text { I: Gait performance } \\
\text { with/without verbal } \\
\text { instruction, verbal } \\
\text { instruction- } \\
\text { metronome cueing, } \\
\text { stepping sound, } \\
\text { stepping sound- } \\
\text { verbal instructions, } \\
\text { for small and wide } \\
\text { stride length } \\
\text { (randomized) } \\
\text { II: Gait performance } \\
\text { with/without } \\
\text { stepping sound, } \\
\text { verbal instruction- } \\
\text { stepping sound } \\
\text { feedback, } \\
\text { synthesized gravel } \\
\text { sound, synthesized } \\
\text { gravel sound-verbal } \\
\text { instructions, for }\end{array}$ & $\begin{array}{l}\text { I: RAC (Ct: } 550- \\
649 \mathrm{~ms}, \text { Exp: } 600- \\
700 \mathrm{~ms}) \text {, foot step } \\
\text { feedback on gravel } \\
\text { (500,600, 700ms) } \\
\text { II: RAC (Ct: } 550- \\
649 \mathrm{~ms}, \text { Exp: } 600- \\
700 \mathrm{~ms}), \text { foot step } \\
\text { feedback on gravel } \\
\text { (500, 600, 700ms), } \\
\text { synthesized gravel step } \\
\text { sound corresponding } \\
\text { to plantar force } \\
\text { (developed by using } \\
\text { ground reaction forces } \\
\text { vector to modulate } \\
\text { both intensity envelop, } \\
\text { and central frequency } \\
\text { of bandpass filter }\end{array}$ & $\begin{array}{l}\text { Significant enhancement in stride length } \\
\text { for healthy Ct in all cueing conditions. } \\
\text { No effect of auditory cueing or } \\
\text { instructions on mean step duration. } \\
\text { Significant reduction in stride length } \\
\text { variability with synthesized feedback as } \\
\text { compared to footstep feedback-verbal } \\
\text { instruction, synthesized feedback-verbal } \\
\text { instructions. } \\
\text { Significant reduction in stride length } \\
\text { variability with stepping, synthesized } \\
\text { feedback, stepping-verbal instructions. } \\
\text { Significant enhancements in stride } \\
\text { length with rhythmic auditory cueing } \\
\text { (synthesized) and motor imagery } \\
\text { together. } \\
\text { No effect on stride duration parameters. }\end{array}$ \\
\hline
\end{tabular}




\begin{tabular}{|c|c|c|c|c|c|c|}
\hline & & & & $\begin{array}{l}\text { small and wide } \\
\text { stride length } \\
\text { (randomized) } \\
\text { III: Gait performance } \\
\text { with/without motor } \\
\text { imagery, motor } \\
\text { imagery-stepping } \\
\text { sound feedback, } \\
\text { synthesized gravel } \\
\text { sound, synthesized } \\
\text { gravel sound-motor } \\
\text { imagery, for small } \\
\text { and wide stride } \\
\text { length (randomized) }\end{array}$ & $\begin{array}{l}\text { applied to stochastic } \\
\text { noise impulse signal) } \\
\text { III: same as II }\end{array}$ & \\
\hline $\begin{array}{l}\text { Leow, et al. } \\
{[105]}\end{array}$ & $\begin{array}{l}24 \mathrm{~F}, \quad 19 \mathrm{M} \\
(18-20)\end{array}$ & 5 & $\begin{array}{l}\text { Stride velocity, step } \\
\text { length, step time, } \\
\text { stride width, double } \\
\text { support, \& } \\
\text { coefficient of } \\
\text { variability for stride } \\
\text { length }\end{array}$ & $\begin{array}{l}\text { Gait performance } \\
\text { with/without } \\
\text { rhythmic music, } \\
\text { RAC (low/high } \\
\text { groove) at } 0 \% \& \\
+22.5 \% \text { of preferred } \\
\text { cadence }\end{array}$ & $\begin{array}{l}\text { RAC (low/high groove } \\
\text { music) at } 0 \% \quad \& \\
+22.5 \% \text { of preferred } \\
\text { cadence }(50 \mathrm{~ms} 1 \mathrm{kHz} \\
\text { sine tones) }\end{array}$ & $\begin{array}{l}\text { Significant enhancement in stride } \\
\text { velocity with rhythmic music cueing } \\
\text { (high groove) \& metronome at }+22.5 \% \text { of } \\
\text { preferred cadence as compared to no } \\
\text { cueing. } \\
\text { Significant reduction in double support } \\
\text { with metronome cueing at } 0 \% \&+25 \% \\
\text { of preferred cadence as compared to no } \\
\text { cueing. } \\
\text { Significant reduction in step length with } \\
\text { high groove music at }+25 \% \text { of preferred } \\
\text { cadence. } \\
\text { Significant reduction in step time in low } \\
\text { (0\% also), high groove music cueing \& } \\
\text { RAC at }+25 \% \text { of preferred cadence } \\
\text { cueing as compared to no cueing. } \\
\text { Significant enhancement in coefficient of } \\
\text { variability for stride length with low \& } \\
\text { high groove RAC at } 0 \% \&+25 \% \text { of } \\
\text { preferred cadence. }\end{array}$ \\
\hline $\begin{array}{l}\text { Sejdić, et al. } \\
{[164]}\end{array}$ & $\begin{array}{l}8 \mathrm{~F}, \quad 7 \mathrm{M} \\
(23.9 \pm 4.7)\end{array}$ & 5 & $\begin{array}{l}\text { Gait speed, mean } \\
\text { stride interval, } \\
\text { stride interval } \\
\text { variability, stride } \\
\text { interval dynamics, } \\
\text { dynamic stability of } \\
\text { gait in anterior- } \\
\text { posterior, vertical \& } \\
\text { medio-lateral } \\
\text { dimension (short: } \\
\text { between } 0^{\text {th }} \& 1^{\text {st }} \\
\text { stride \& long: } \\
\text { between } 4^{\text {th }} \& 10^{\text {th }} \\
\text { stride, term } \\
\text { Lyapunov } \\
\text { exponent) }\end{array}$ & $\begin{array}{l}\begin{array}{l}\text { Gait performance } \\
\text { with }\end{array} \\
\text { auditory, visual \& } \\
\text { haptic cueing } \\
\text { (randomly spate or } \\
\text { together) at preferred } \\
\text { cadence during } 2 \\
\text { sessions }\end{array}$ & $\begin{array}{l}\text { RAC at preferred } \\
\text { cadence }\end{array}$ & $\begin{array}{l}\text { Significantly reduced stride interval } \\
\text { variability with RAC (alone \& combined } \\
\text { with visual \& haptic cueing) as compared } \\
\text { to no cueing condition. } \\
\text { Significantly reduced stride interval } \\
\text { dynamics (long term Lyapunov } \\
\text { exponent) with RAC (alone \& combined } \\
\text { with visual \& haptic cueing) as compared } \\
\text { to no cueing condition. } \\
\text { Significant enhancement in dynamic } \\
\text { stability of gait with RAC (alone \& } \\
\text { combined with visual \& haptic cueing) as } \\
\text { compared to no cueing condition. }\end{array}$ \\
\hline $\begin{array}{l}\text { Terrier and } \\
\text { Dériaz [165] }\end{array}$ & $\begin{array}{l}10 \mathrm{~F}, \quad 10 \mathrm{M} \\
(36 \pm 11)\end{array}$ & 4 & $\begin{array}{l}\text { DFA on time series } \\
\text { of stride time, stride } \\
\text { length \& stride } \\
\text { speed } \\
\text { Short \& long-term } \\
\text { local dynamic } \\
\text { stability in anterior- } \\
\text { posterior \& medial- } \\
\text { lateral direction }\end{array}$ & $\begin{array}{l}\text { Gait performance on } \\
\text { treadmill at slow }(0.7 \\
\text { times preferred } \\
\text { cadence), fast }(1.3 \\
\text { times preferred } \\
\text { cadence) \& at } \\
\text { preferred cadence } \\
\text { with/ without RAC } \\
\text { (randomly) }\end{array}$ & $\begin{array}{l}\text { RAC at slow ( } 0.7 \text { times } \\
\text { slower than preferred } \\
\text { cadence), fast }(1.3 \\
\text { times faster than } \\
\text { preferred cadence }) \\
\text { cadence }\end{array}$ & $\begin{array}{l}\text { Significant enhancement in long term } \\
\text { local dynamic stability with RAC } \\
\text { Significant reduction of stride time \& } \\
\text { stride length variability at slow speed } \\
\text { with RAC } \\
\text { No effect on short term local dynamic } \\
\text { stability with RAC }\end{array}$ \\
\hline $\begin{array}{l}\text { Roerdink, et } \\
\text { al. [166] }\end{array}$ & $\begin{array}{l}10 \mathrm{~F}, \quad 10 \mathrm{M} \\
(63.2 \pm 3.6)\end{array}$ & 5 & $\begin{array}{l}\text { Cadence, mean } \\
\text { relative timing } \\
\text { between footfalls \& } \\
\text { auditory stimuli, } \\
\text { variability of mean } \\
\text { relative timing (by } \\
\text { circular statistics) }\end{array}$ & $\begin{array}{l}\text { Participants } \\
\text { performed gait at } \\
\text { preferred cadence } \\
\text { followed by } 7 \\
\text { random trials with } \\
\text { adjusted RAC i.e. } \\
77.5 \%, 85 \%, 92.5 \% \text {, } \\
100 \% \text {, } 107.5 \% \text {, } \\
115 \% \text { or } 122.5 \%\end{array}$ & $\begin{array}{l}\text { Auditory input from } \\
\text { drum RAC at } 77.5 \% \text {, } \\
85 \%, 92.5 \%, 100 \% \text {, } \\
107.5 \%, 115 \% \text { or } \\
122.5 \% \text { of preferred } \\
\text { cadence } \\
\text { Different pitch to pace } \\
\text { for RAC i.e. for step } \\
\text { left: } 440 \mathrm{~Hz} \text { right: } \\
1000 \mathrm{~Hz}\end{array}$ & $\begin{array}{l}\text { Significant effect of RAC on cadence, } \\
\text { mean relative timing \& variability of } \\
\text { mean relative timing between footfalls \& } \\
\text { auditory inputs. } \\
\text { Significantly fewer steps required to } \\
\text { reach synchronization }\end{array}$ \\
\hline $\begin{array}{l}\text { Lohnes and } \\
\text { Earhart [67] }\end{array}$ & $\begin{array}{l}\text { Young: } 7 \mathrm{~F}, \\
4 \mathrm{M} \\
(24 \pm 0.8) \\
\text { Old: } 7 \mathrm{~F}, 4 \mathrm{M} \\
(70.8 \pm 10.4)\end{array}$ & 5 & 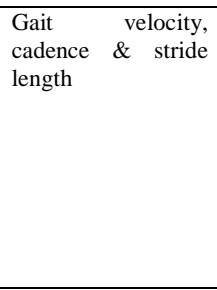 & $\begin{array}{l}\text { Patients performed } \\
\text { gait with/without } \\
\text { RAC at }-10 \%,+10 \% \\
\text { of preferred cadence } \\
\text { or with additional } \\
\text { cueing strategy } \\
\text { "think about larger } \\
\text { strides" with/without } \\
-10 \% \text { \& }+10 \% \text { of } \\
\text { auditory inputs tone, }\end{array}$ & $\begin{array}{l}\text { RAC at } \pm 10 \% \text { of } \\
\text { preferred cadence. }\end{array}$ & $\begin{array}{l}\text { Significant effect on gait velocity stride } \\
\text { length, cadence for both groups with } \\
\pm 10 \% \text { of RAC under both single and } \\
\text { dual-task conditions. } \\
\text { Larger effects noted in young } \\
\text { participants as compared to older } \\
\text { counterparts. } \\
\text { Verbal instructions had no influence on } \\
\text { cadence among both groups under both } \\
\text { single and dual-task conditions. }\end{array}$ \\
\hline
\end{tabular}




\begin{tabular}{|c|c|c|c|c|c|c|}
\hline & & & & $\begin{array}{l}\text { with/without dual- } \\
\text { task "word } \\
\text { generation task" }\end{array}$ & & \\
\hline $\begin{array}{l}\text { Trombetti, et } \\
\text { al. [167] }\end{array}$ & $\begin{array}{l}\text { Exp: } \quad 64 \mathrm{~F}, \\
2 \mathrm{M}(75 \pm 8) \\
\mathrm{Ct}: 65 \mathrm{~F}, 3 \mathrm{M} \\
(76 \pm 6)\end{array}$ & 8 & $\begin{array}{l}\text { Gait velocity, stride } \\
\text { length, cadence, } \\
\text { double, single } \\
\text { support phase, } \\
\text { stride time/length } \\
\text { variability, TUG } \\
\text { test, trunk angular } \\
\text { displacement, } \\
\text { Tinetti tests \& } \\
\text { assessment of falls }\end{array}$ & $\begin{array}{l}\text { Exp: Pre-test, gait \& } \\
\text { exercise training } \\
\text { with auditory input } \\
\text { performed for 1-hour } \\
\text { session/ week for } 12 \\
\text { months, 6-month } \\
\text { test, post-test, } \\
\text { with/without dual- } \\
\text { task (counting } \\
\text { backward aloud task) } \\
\text { Ct: started 6-month } \\
\text { delayed intervention, } \\
\text { with/without dual- } \\
\text { task (counting } \\
\text { backward aloud task) }\end{array}$ & $\mathrm{RAC}$ as piano music & $\begin{array}{l}\text { Single task: Significant enhancement in } \\
\text { gait velocity, stride length \& stride time } \\
\text { variability for the Exp as compared to Ct. } \\
\text { Dual-task: Significant enhancement in } \\
\text { stride length, decrease in stride length } \\
\text { variability in Exp as compared to Ct } \\
\text { Significant enhancement in } 1 \text { legged } \\
\text { stance, Tinetti tests, TUG \& decreased } \\
\text { mediolateral angular velocity. } \\
\text { Significantly reduced incidences of falls } \\
\text { in Exp as compared to Ct. }\end{array}$ \\
\hline $\begin{array}{l}\text { Wittwer, et } \\
\text { al. [136] }\end{array}$ & $\begin{array}{l}12 \mathrm{~F}, \quad 7 \mathrm{M} \\
(79 \pm 7.8)\end{array}$ & 4 & $\begin{array}{l}\text { Swing time, stride } \\
\text { time, velocity, stride } \\
\text { length, double } \\
\text { support \%, stride } \\
\text { width, stride length } \\
\text { \& time variability }\end{array}$ & $\begin{array}{l}\text { Participants } \\
\text { performed gait } \\
\text { with/without } \\
\text { auditory feedback } \\
\text { "randomly" i.e. } \\
\text { music or RAC }\end{array}$ & $\begin{array}{l}\text { Music or metronome or } \\
\text { RAC at participants } \\
\text { preferred cadence }\end{array}$ & $\begin{array}{l}\text { Significant enhancement in velocity, } \\
\text { stride length with music as compared to } \\
\text { no sound. } \\
\text { Significant reduction in stride time, } \\
\text { double limb support \& enhancement in } \\
\text { cadence with both music \& RAC input, } \\
\text { as compared to no auditory input. } \\
\text { No effect on mean step width, mean } \\
\text { temporal or spatial gat variability. }\end{array}$ \\
\hline Yu, et al. [93] & $\begin{array}{l}13 \mathrm{~F} \\
(21.8 \pm 0.4)\end{array}$ & 5 & $\begin{array}{l}\text { Stride length, } \\
\text { cadence \& } \begin{array}{l}\text { gait } \\
\text { speed }\end{array}\end{array}$ & $\begin{array}{l}\text { Gait performance } \\
\text { with/without RAC at } \\
0 \% \& \pm 10 \% \text { of } \\
\text { preferred cadence }\end{array}$ & $\begin{array}{l}\text { RAC at } 0 \% \& \pm 10 \% \text { of } \\
\text { preferred cadence }\end{array}$ & $\begin{array}{l}\text { Significant enhancement in stride length, } \\
\text { cadence \& gait speed with }+10 \% \text { RAC as } \\
\text { compared to all conditions. } \\
\text { Significant reduction in cadence \& gait } \\
\text { speed with }-10 \% \text { of RAC as compared to } \\
0 \% \text { \& no cueing. }\end{array}$ \\
\hline $\begin{array}{l}\text { Almeida, et } \\
\text { al. [92] }\end{array}$ & $\begin{array}{l}\text { Exp I: } 9 \\
(42.7 \pm 6.6) \\
\text { Exp II: } 10 \\
(42.4 \pm 4.5) \\
\text { Ct: } 9 \\
(41.7 \pm 5)\end{array}$ & 4 & $\begin{array}{l}\text { Gait speed, heart } \\
\text { rate, maximal } \\
\text { oxygen } \\
\text { consumption, rating } \\
\text { of perceived } \\
\text { exertion }\end{array}$ & $\begin{array}{l}\text { Gait performance } \\
\text { with/without (Ct) } \\
\text { RAC at } 90 \mathrm{bpm}(\operatorname{Exp} \\
\text { II) \& } 140 \mathrm{bpm}(\operatorname{Exp} \\
\text { I) for } 30 \text { minutes } \\
\text { with re-tests at every } \\
\text { 5-minute interval }\end{array}$ & $\mathrm{RAC}$ at $90 \& 140 \mathrm{bpm}$ & $\begin{array}{l}\text { Significant enhancement in gait } \\
\text { performance in Exp I as compared to Exp } \\
\text { II \& Ct. } \\
\text { No effect on heart rate \& maximal } \\
\text { oxygen consumption in Exp or Ct. }\end{array}$ \\
\hline $\begin{array}{l}\text { Hunt, } \\
\text { McGrath and } \\
\text { Stergiou } \\
{[168]}\end{array}$ & $\begin{array}{l}4 \mathrm{~F}, \quad 6 \mathrm{M} \\
(28.1 \pm 5.3)\end{array}$ & 4 & $\begin{array}{l}\text { Stride time, sample } \\
\text { entropy of stride } \\
\text { time interval for } \\
\text { individualized } \\
\text { fractal RAC, DFA } \\
\text { for auditory signals } \\
\text { scaling exponent \& } \\
\text { stride time scaling } \\
\text { exponent }\end{array}$ & $\begin{array}{l}\text { Gait performance } \\
\text { with/without } \\
\text { individualized } \\
\text { fractal RAC for } \\
\text { white, pink \& brown } \\
\text { noise (randomized) }\end{array}$ & 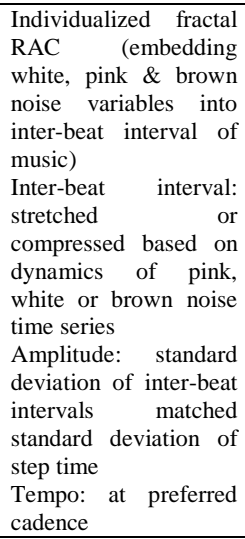 & $\begin{array}{l}\text { Significant effect of RAC on sample } \\
\text { entropy of stride interval time series } \\
\text { (brown>pink> white }>\text { no sound) } \\
\text { Significant enhancement of fractal } \\
\text { scaling exponent with auditory feedback } \\
\text { of stride interval time series } \\
\text { (brown>pink }>\text { white>no sound) }\end{array}$ \\
\hline \multirow[t]{2}{*}{$\begin{array}{l}\text { Marmelat, et } \\
\text { al. [169] }\end{array}$} & $7 F(28 \pm 6)$ & 5 & $\begin{array}{l}\text { DFA of inter slide } \\
\text { interval variability, } \\
\text { inter-beat interval } \\
\text { variability \& \& } \\
\text { asynchrony with } \\
\text { metronome between } \\
\text { two successive right } \\
\text { heel strikes }\end{array}$ & $\begin{array}{l}\text { Gait performed on } \\
\text { treadmill with } \\
\text { /without RAC with } \\
\text { either IC or fractal } \\
\text { feedback }\end{array}$ & $\begin{array}{l}\text { RAC with either IC or } \\
\text { fractal feedback } \\
\text { Inter-beat intervals } \\
\text { contained fractal } \\
\text { Gaussian noise with } \\
\text { corresponding scaling } \\
\text { exponent }(600 \mathrm{~Hz})\end{array}$ & $\begin{array}{l}\text { Significant effects of pacing rhythmic } \\
\text { metronome feedback on global } \\
\text { exponents of inter-beat \& slide intervals } \\
\text { (persistent correlations) } \\
\text { No effect on inter slide interval, } \\
\text { asynchrony with RAC } \\
\text { Participants anticipated the metronome } \\
\& \text { adapted with pacing stimuli } \\
\text { No significant correlations between } \\
\text { inter-beat intervals \& inter-slide intervals } \\
\text { (increased correlation with increased } \\
\text { variability) }\end{array}$ \\
\hline & $\begin{array}{ll}5 \mathrm{~F}, & 7 \mathrm{M} \\
(28 \pm 6) & \end{array}$ & 5 & $\begin{array}{l}\text { DFA of inter slide } \\
\text { interval variability, } \\
\text { inter-beat interval } \\
\text { variability \& } \\
\text { asynchrony with } \\
\text { metronome between } \\
\text { two successive right } \\
\text { heel strikes }\end{array}$ & $\begin{array}{l}\text { Gait performed on } \\
\text { treadmill with } \\
\text { /without RAC with } \\
\text { either IC or fractal } \\
\text { feedback }\end{array}$ & $\begin{array}{ll}\text { RAC with } & \text { non-IC } \\
\text { (different } & \text { scaling } \\
\text { exponents) } & \end{array}$ & $\begin{array}{l}\text { Significant effects of pacing rhythmic } \\
\text { metronome feedback on global } \\
\text { exponents of inter-slide intervals (anti- } \\
\text { persistent correlations) } \\
\text { No significant correlations between } \\
\text { inter-beat intervals \& interslide intervals } \\
\text { (increased correlation with increased } \\
\text { variability) }\end{array}$ \\
\hline
\end{tabular}




\begin{tabular}{|c|c|c|c|c|c|c|}
\hline $\begin{array}{l}\text { Franěk, et al. } \\
\text { [68] }\end{array}$ & $\begin{array}{l}30 \mathrm{~F}, \quad 42 \mathrm{M} \\
(20.2 \pm 1.2)\end{array}$ & 4 & $\begin{array}{l}\text { Gait speed, } \\
\text { synchronization } \\
\text { (inter step times) }\end{array}$ & $\begin{array}{l}\text { Gait performed } \\
\text { with/without } \\
\text { rhythmic music } \\
\text { feedback at } 114,124, \\
133 \text { bpm }\end{array}$ & $\begin{array}{l}\text { RAC at } 114,124,133 \\
\text { bpm }\end{array}$ & $\begin{array}{l}\text { Significant enhancement in gait speed } \\
\text { with faster tempo music feedback as } \\
\text { compared to slower tempo RAC \& no } \\
\text { feedback. } \\
\text { No effect on synchronization with } \\
\text { rhythmic music feedback. }\end{array}$ \\
\hline & $\begin{array}{l}60 \mathrm{~F}, \quad 61 \mathrm{M} \\
(20.6 \pm 1.5)\end{array}$ & 4 & $\begin{array}{l}\text { Gait speed, } \\
\text { synchronization } \\
\text { (inter step times) }\end{array}$ & $\begin{array}{l}\text { Gait performed } \\
\text { with/without] RAC } \\
\text { (music } \\
\text { motivational/non- } \\
\text { motivational) }\end{array}$ & $\begin{array}{lr}\text { RAC } & \text { (music } \\
\text { motivational: } & 131-200 \\
\text { bpm, } & \text { non- } \\
\text { motivational: } & 52-96 \\
\text { bpm) } & \\
\end{array}$ & $\begin{array}{l}\text { Significant enhancement in gait speed } \\
\text { with motivational rhythmic music } \\
\text { feedback as compared to non- } \\
\text { motivational RAC \& no feedback. }\end{array}$ \\
\hline $\begin{array}{l}\text { Leman, et al. } \\
{[142]}\end{array}$ & $\begin{array}{ll}11 \mathrm{~F}, & 7 \mathrm{M} \\
(22-51) & \end{array}$ & 4 & $\begin{array}{l}\text { Gait speed, gait } \\
\text { tempo, } \\
\text { synchronization of } \\
\text { steps to tempo }\end{array}$ & 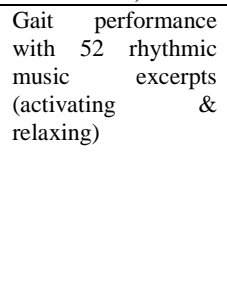 & $\begin{array}{l}\text { RAC (relaxing or } \\
\text { activating effects) at } \\
130 \text { beats per minute, } \\
\text { short fade in of } 50 \mathrm{~ms} \\
\& \text { fade out of } 100 \mathrm{~ms} \\
\text { applied to each musical } \\
\text { excerpt } \\
\text { RAC superimposed at } \\
\text { position } 1,12,23,34, \\
45, \& 58\end{array}$ & $\begin{array}{l}\text { Significant effect of activating (increased } \\
\text { gait speed), relaxing (reduced gait speed) } \\
\text { in gait speed with RAC with same tempo. } \\
\text { Significant enhancement } \\
\text { synchronization of steps with RAC }\end{array}$ \\
\hline $\begin{array}{l}\text { Peper, et al. } \\
{[170]}\end{array}$ & $\begin{array}{l}\text { Young: } 4 \mathrm{~F} \\
8 \mathrm{M}(22-28) \\
\text { Old: } 5 \mathrm{~F}, 7 \mathrm{M} \\
(55-69)\end{array}$ & 5 & $\begin{array}{l}\text { Mean reaction time, } \\
\text { gait speed, step } \\
\text { length, step width }\end{array}$ & $\begin{array}{lr}\text { Gait } & \text { performed } \\
\text { with/without RAC \& } \\
\text { visual } & \text { feedback } \\
\text { (stepping } & \text { stones), } \\
\text { dual-task } & \text { (probe } \\
\text { reaction } & \text { task } \\
\text { generating } & \text { vibrating } \\
\text { stimuli) } & \\
\end{array}$ & $\begin{array}{l}\text { RAC Left }(440 \mathrm{~Hz}), \\
\text { right }(1000 \mathrm{~Hz}) \\
\text { Temporal shift of } \\
\pm 1 / 6^{\text {th }} \text { of interval } \\
\text { between consecutive } \\
\text { ipsilateral beeps, } \\
\text { causing } \pm 60^{\circ} \text { phase } \\
\text { delay/advance }\end{array}$ & $\begin{array}{l}\text { Significantly enhanced step length \& step } \\
\text { width RAC No effect on gait speed in } \\
\text { young \& older adults with RAC } \\
\text { Significantly enhanced reaction times } \\
\text { with RAC as compared to no cueing. } \\
\text { Significantly reduced reaction time with } \\
\text { RAC as compared to visual cueing. }\end{array}$ \\
\hline $\begin{array}{l}\text { Bank, } \\
\text { Roerdink } \\
\text { and Peper } \\
{[171]}\end{array}$ & $\begin{array}{l}10 \mathrm{~F}, \quad 10 \mathrm{M} \\
(63.2 \pm 3.6)\end{array}$ & 5 & $\begin{array}{l}\text { Mean normalized } \\
\text { step time, step } \\
\text { length, relative } \\
\text { phase shift between } \\
\text { gait \& cues }\end{array}$ & $\begin{array}{l}\text { Gait performance } \\
\text { with RAC } \pm 22.5 \% \\
\text { (introduced in steps } \\
\text { of } \pm 7.5 \% \text { randomly) } \\
\text { of preferred cadence } \\
\text { \&/or stepping stone } \\
\text { visual feedback }\end{array}$ & $\begin{array}{l}\text { RAC at } \pm 22.5 \% \text { of } \\
\text { preferred cadence } \\
\text { Temporal shift of } \\
\pm 1 / 6^{\text {th }} \text { of interval } \\
\text { between consecutive } \\
\text { ipsilateral beeps, } \\
\text { causing } \pm 60^{\circ} \text { phase } \\
\text { delay/advance }\end{array}$ & $\begin{array}{l}\text { Significant effect of phase delay on } \\
\text { increasing/decreasing step length, step } \\
\text { time with auditory \& visual feedback. } \\
\text { However visual cueing > RAC } \\
\text { Significantly enhanced phase shift from } \\
\text { auditory to visual cueing condition. } \\
\text { Significant reduction in coordination of } \\
\text { RAC with gait as compared to visual } \\
\text { cueing }\end{array}$ \\
\hline $\begin{array}{l}\text { Wellner, et } \\
\text { al. [91] }\end{array}$ & $17(28 \pm 8)$ & 4 & $\begin{array}{lr}\text { Obstacle } & \text { hit } \%, \\
\text { average } & \text { obstacle } \\
\text { clearance } & \& \\
\text { individually } & \text { chosen } \\
\text { gait speed } & \end{array}$ & $\begin{array}{l}\text { Gait performance on } \\
\text { robot assisted device } \\
\text { with/without } \\
\text { Rhythmic auditory } \\
\text { feedback (distance to } \\
\text { obstacle \&/or foot } \\
\text { clearance feedback) }\end{array}$ & $\begin{array}{l}\text { Rhythmic real-time } \\
\text { feedback for distance } \\
\text { to obstacle \& foot } \\
\text { clearance } \\
\text { Obstacle distance: } \\
\text { Rhythm (repeating } \\
\text { sound with shorter } \\
\text { pause interval as } \\
\text { distance decreases), } \\
\text { continuous/discrete } \\
\text { pitch (continuous } \\
\text { sound with higher pitch } \\
\text { as distance } \\
\text { increases/decreases), } \\
\text { dynamics (increase in } \\
\text { volume as distance } \\
\text { decreases) fors } \\
\text { Absolute harmony } \\
\text { clearance: harmony } \\
\text { (dissonant/consonant } \\
\text { chords below/above } \\
\text { obstacle), pitch with } 2 \\
\& 3 \text { levels, noise } \\
\text { (Gaussian noise below, } \\
\text { no sound above } \\
\text { obstacle) }\end{array}$ & $\begin{array}{l}\text { Significantly enhanced self-chosen gait } \\
\text { speed with auditory feedback as } \\
\text { compared to only visual feedback. } \\
\text { Significant enhancement in gait speed } \\
\text { with rhythmic feedback for distance to } \\
\text { obstacle \&/or foot clearance as compared } \\
\text { to no feedback }\end{array}$ \\
\hline $\begin{array}{l}\text { Arias and } \\
\text { Cudeiro } \\
{[102]}\end{array}$ & $\begin{array}{l}6 \mathrm{~F}, \quad 5 \mathrm{M} \\
(65.7 \pm 7.6)\end{array}$ & 5 & $\begin{array}{l}\text { Cadence, } \\
\text { velocity, } \\
\text { amplitude, } \\
\text { coefficient } \\
\text { variation for } \\
\text { amplitude \& } \text { step } \\
\text { time }\end{array}$ & $\begin{array}{l}\text { Patients performed } \\
\text { gait with/without } \\
\text { rhythmic cueing } \\
\text { from auditory, visual } \\
\& \text { audio-visual } \\
\text { condition, with } \\
\text { frequency ranging } \\
\text { from } 70-110 \% \\
\text { increment/decrement } \\
\text { at } \pm 10 \% \text { of preferred } \\
\text { cadence }\end{array}$ & $\begin{array}{l}\text { RAC with wave } \\
\text { frequency of } 4625 \mathrm{~Hz} \\
\text { delivered at frequency } \\
\text { ranging from } 70-110 \% \\
\text { increment/decrement } \\
\text { at } \pm 10 \% \text { of preferred } \\
\text { cadence }\end{array}$ & $\begin{array}{l}\text { Significant enhancement in cadence, step } \\
\text { amplitude in Ct with RAC } \\
\text { No effects on gait velocity, coefficient of } \\
\text { variability for stride time \& stride } \\
\text { amplitude. }\end{array}$ \\
\hline $\begin{array}{l}\text { Baker, et al. } \\
{[172]}\end{array}$ & $\begin{array}{l}7 \mathrm{~F}, \quad 5 \mathrm{M} \\
(71.5 \pm 2.5)\end{array}$ & 7 & $\begin{array}{lr}\text { Gait } & \text { speed, } \\
\text { coefficient } & \text { of } \\
\text { velocity for } & \text { (step }\end{array}$ & $\begin{array}{l}\text { Pre-test, functional } \\
\text { gait performance } \\
\text { with/without RAC - } \\
10 \% \text { of preferred }\end{array}$ & $\begin{array}{l}\text { RAC at }-10 \% \text { of } \\
\text { preferred cadence }\end{array}$ & $\begin{array}{l}\text { Significant effect of RAC back and } \\
\text { verbal instructions on enhancing stride } \\
\text { length, gait velocity. }\end{array}$ \\
\hline
\end{tabular}




\begin{tabular}{|c|c|c|c|c|c|c|}
\hline & & & $\begin{array}{l}\text { time, double limb } \\
\text { support time) }\end{array}$ & $\begin{array}{l}\text { cadence, attentional } \\
\text { cue instructions "try } \\
\text { to take big steps", } \\
\text { together "take a big } \\
\text { step with the beat", } \\
\& \text { with/without a } \\
\text { dual-task (a tray with } \\
2 \text { cups of water on } \\
\text { top), post-test }\end{array}$ & & $\begin{array}{l}\text { Significantly reduced cadence with RAC } \\
\text { and verbal instructions. } \\
\text { Reduced gait speed, cadence with }-10 \% \\
\text { RAC No effect on stride length. }\end{array}$ \\
\hline $\begin{array}{l}\text { Hausdorff, et } \\
\text { al. [117] }\end{array}$ & $\begin{array}{l}14 \mathrm{~F}, \quad 12 \mathrm{M} \\
(64.6 \pm 6.8)\end{array}$ & 5 & $\begin{array}{l}\text { Stride time, gait } \\
\text { speed, stride length, } \\
\text { swing time, stride } \\
\text { time variability \& } \\
\text { swing time } \\
\text { variability }\end{array}$ & $\begin{array}{l}\text { Pre-test, gait } \\
\text { performance } \\
\text { with/without RAC at } \\
\text { preferred cadence, } \\
+10 \%, \text { Post-test } 2 \& \\
15 \text { min short term } \\
\text { retention test }\end{array}$ & $\begin{array}{l}\text { RAC at } 0 \% \&+10 \% \text { of } \\
\text { preferred cadence }\end{array}$ & $\begin{array}{l}\text { Significant enhancement in gait speed } \\
\text { with }+10 \% \text { RAC } \\
\text { Significant reduction in stride time with } \\
+10 \% \text { RAC } \\
\text { No effect on stride length, swing time, } \\
\text { stride time variability, swing time } \\
\text { variability with RAC }\end{array}$ \\
\hline $\begin{array}{l}\text { Willems, et } \\
\text { al. }[103]\end{array}$ & $9(68.1 \pm 7.3)$ & 5 & $\begin{array}{l}\text { Steps (number, } \\
\text { time, height, width, } \\
\text { length), step length, } \\
\text { step width, step } \\
\text { duration, coefficient } \\
\text { of variation of step } \\
\text { duration }\end{array}$ & $\begin{array}{lr}\text { Gait } & \text { performance } \\
\text { while } & \text { turning } \\
\text { with/without RAC }\end{array}$ & $\begin{array}{l}\text { RAC at preferred } \\
\text { cadence }\end{array}$ & $\begin{array}{l}\text { Enhancement in step length. } \\
\text { No effects on steps (number, time, } \\
\text { height, width), step length, step width, } \\
\text { step duration, coefficient of variation of } \\
\text { step duration with RAC }\end{array}$ \\
\hline $\begin{array}{l}\text { Baram and } \\
\text { Miller [99] }\end{array}$ & $\begin{array}{l}6 \mathrm{~F}, \quad 5 \mathrm{M} \\
(25.4 \pm 1.9)\end{array}$ & 4 & $\begin{array}{l}\text { Gait speed, stride } \\
\text { length, } 10 \text { meters } \\
\text { walking test }\end{array}$ & $\begin{array}{l}\text { Pre-test, followed by } \\
\text { rhythmic auditory } \\
\text { feedback \& } 10 \text { min } \\
\text { follow-up short term } \\
\text { residual performance } \\
\text { test }\end{array}$ & $\begin{array}{l}\text { Rhythmic auditory } \\
\text { feedback generated } \\
\text { with gait step in real- } \\
\text { time }\end{array}$ & $\begin{array}{l}\text { No effects on stride length and gait } \\
\text { velocity with rhythmic feedback } \\
\text { generated in real-time }\end{array}$ \\
\hline $\begin{array}{l}\text { Willems, et } \\
\text { al. [173] }\end{array}$ & $\begin{array}{l}10 \\
(67.2 \pm 9.1)\end{array}$ & 4 & $\begin{array}{l}\text { Step frequency, gait } \\
\text { speed, stride length } \\
\& \text { double support } \\
(\%) \text { phase }\end{array}$ & $\begin{array}{l}\text { Pre-test, } \\
\text { performance at } 0 \%,- \\
20 \%,-10 \%,+10 \%, \\
+20 \% \text { of RAC } \\
\text { (randomized), post- } \\
\text { test }\end{array}$ & $\begin{array}{l}\text { RAC at } 0 \%,-20 \%,- \\
10 \%,+10 \%,+20 \% \\
\text { preferred cadence }\end{array}$ & $\begin{array}{l}\text { Significant effect of RAC on cadence, } \\
\text { gait speed, with } 0 \%,-10 \%,+10 \%,+20 \% \\
\text { pacing of RAC } \\
\text { No significant effects on double limb } \\
\text { support, stride length }\end{array}$ \\
\hline $\begin{array}{l}\text { Baker, et al. } \\
{[101]}\end{array}$ & $\begin{array}{l}7 \mathrm{~F}, \quad 4 \mathrm{M} \\
(71.5 \pm 2.5)\end{array}$ & 6 & $\begin{array}{l}\text { Gait speed, step } \\
\text { amplitude \& step } \\
\text { frequency }\end{array}$ & $\begin{array}{l}\text { Pre-test, functional } \\
\text { gait performance } \\
\text { with/without RAC - } \\
10 \% \text { of preferred } \\
\text { cadence, attentional } \\
\text { cue instructions "try } \\
\text { to take big steps", } \\
\text { together "take a big } \\
\text { step with the beat", } \\
\& \text { with/without a } \\
\text { dual-task (a tray with } \\
2 \text { cups of water on } \\
\text { top), post-test }\end{array}$ & $\begin{array}{l}\text { RAC at }-10 \% \text { of } \\
\text { preferred cadence }\end{array}$ & $\begin{array}{l}\text { Significant effect of RAC \& attentional } \\
\text { cue "big steps with beat" on step } \\
\text { frequency in gait speed (single-task } \\
\text { only), step amplitude, step frequency in } \\
\mathrm{Ct} \text { in both single \& dual-task conditions } \\
\text { Non-significant effects on gait speed, } \\
\text { step amplitude \& step frequency with } \\
\text { RAC only. } \\
\text { Effects not evitable once the RAC was } \\
\text { removed, in post-test }\end{array}$ \\
\hline $\begin{array}{l}\text { Rochester, et } \\
\text { al. [94] }\end{array}$ & $\begin{array}{l}4 \mathrm{~F}, \quad 6 \mathrm{M} \\
(63.5 \pm 7)\end{array}$ & 6 & $\begin{array}{l}\text { Step length, step } \\
\text { frequency, walking } \\
\text { speed, time duration } \\
\& \text { cadence }\end{array}$ & $\begin{array}{lr}\text { Complex functional } \\
\text { walking \& sitting } \\
\text { task under single \& } \\
\text { dual-motor task } \\
\text { (carrying a tray) } \\
\text { condition } \\
\text { with/without RAC } \\
\end{array}$ & $\begin{array}{lr}\text { RAC generated per } \\
\text { preferred speed of } \\
\text { patients. }\end{array}$ & $\begin{array}{l}\text { No effects of RAC on gait speed, step } \\
\text { length \& cadence under single/dual-task } \\
\text { conditions. However, reduction in } \\
\text { cadence under dual-task conditions with } \\
\text { RAC }\end{array}$ \\
\hline $\begin{array}{l}\text { Thaut, et al. } \\
{[174]}\end{array}$ & $\begin{array}{l}10 \mathrm{~F}, \\
(25-40)\end{array}$ & 4 & $\begin{array}{l}\text { Stride symmetry, } \\
\text { stride duration \& } \\
\text { EMG amplitude } \\
\text { variability } \\
\text { (Gastrocnemius) }\end{array}$ & 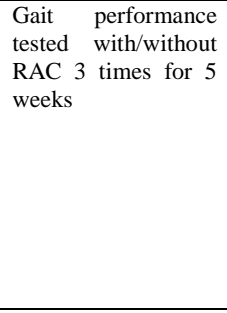 & $\begin{array}{l}\text { RAC at } 4 / 4 \text {-time } \\
\text { signature }\left(1^{\text {st }} \& 3^{\text {rd }} \text { beat }\right. \\
\text { accentuated } \\
\text { tambourine by } \\
\begin{array}{l}70 \mathrm{~dB}) \text { at peat, } \\
\text { cadence, at slower, } \\
\text { faster than preferred } \\
\text { cadence }\end{array}\end{array}$ & $\begin{array}{l}\text { Significant enhancement in stride } \\
\text { rhythmicity between right \& left limb } \\
\text { with RAC } \\
\text { Significantly delayed \& shortened onset } \\
\text { of gastrocnemius EMG activity with } \\
\text { RAC } \\
\text { Significant reduction in EMG variability } \\
\text { of gastrocnemius muscle with RAC } \\
\text { Significantly enhanced integrated } \\
\text { amplitude ratios for gastrocnemius EMG } \\
\text { activity }\end{array}$ \\
\hline $\begin{array}{l}\text { McIntosh, et } \\
\text { al. [175] }\end{array}$ & $\begin{array}{l}6 \mathrm{~F}, \\
(72 \pm 5)\end{array}$ & 4 & $\begin{array}{l}\text { Gait velocity, stride } \\
\text { length, cadence \& } \\
\text { cadence-auditory } \\
\text { stimulus } \\
\text { synchronization }\end{array}$ & $\begin{array}{l}\text { Gait performance by } \\
\text { participants with pre- } \\
\text { test, with \& without } \\
\text { RAC at }+10 \% \text { of } \\
\text { preferred cadence, } \\
\text { post-test }\end{array}$ & $\begin{array}{l}\text { RAC at } 0 \%,+10 \% \text { of } \\
\text { preferred cadence }\end{array}$ & $\begin{array}{l}\text { Significant enhancement in gait velocity } \\
\text { and cadence with RAC } \\
\text { Enhancement in stride length. } \\
\text { No effect on gait symmetry }\end{array}$ \\
\hline
\end{tabular}

F: Female, M: Male, Exp: Experimental group, Ct: Control group, RAC: Rhythmic auditory cueing, DFA: Detrended Fluctual Analysis, PD: Parkinson's disease, EMG: Electromyography, IC: Isosynchronous cueing, bpm: beats per minute. 


\section{Participants}

A total of 854 participants were analyzed in the incorporated studies. Studies were then categorized into sub-groups for evaluating young and elderly participants. Three studies compared the effects of rhythmic auditory cueing amongst young and elderly participants. Eighteen studies evaluated elderly participants $(68 \pm 5.6$ years). A total of 355 participants were evaluated ( 235 females/ 100 males). Two studies did not specify the gender of the participants. All the studies evaluated a mixed gender sample size. Nineteen studies evaluated young participants (26.8 \pm 6 years). A total of 499 participants were evaluated ( 215 females/ 248 males). Two studies did not specify the gender of the included participants [91, 92]. Only one study evaluated a non-mixed gender sample i.e. only females [93]. Descriptive statistics relating to the age (mean \pm standard deviation) of the participants were tabulated across the studies (Table 1).

\section{Risk of bias}

The review included studies scoring $\geq 4$ on PEDro to reduce the incidence of biasing. Moreover, the limitation of research protocols to be included in the review was limited to gold standard randomized controlled trials, cluster randomized controlled trials and controlled clinical trials. The individual scores attained by the studies using the PEDro scale have been reported (Table 1, Supplementary table 2). The average PEDro score for the fifty included studies was computed to be 4.7 out of 10 , indicating fair-quality of the overall studies. One study scored 8 , four scored 6 , fourteen studies scored 5 , and sixteen studies scored 4. Publication bias was analyzed by plotting a Hedge's g against standard error (Fig. 2). Asymmetries concerning mean in the funnel plot might suggest bias (either positive or negative), in which case results are published. Risk of bias across the studies has been demonstrated in Fig. 3.

\section{Meta-Analysis}

\section{$\underline{\text { Outcomes }}$}

The results suggest evidence for a positive impact of rhythmic auditory cueing on spatiotemporal gait parameters amongst both young and elderly participants. In the included thirty-four studies, thirty studies reported significant enhancements, two studies reported enhancements ( $p>0.05)$ [94, 95], and two studies reported significant reduction in gait parameters with rhythmic auditory cueing $[96,97]$.

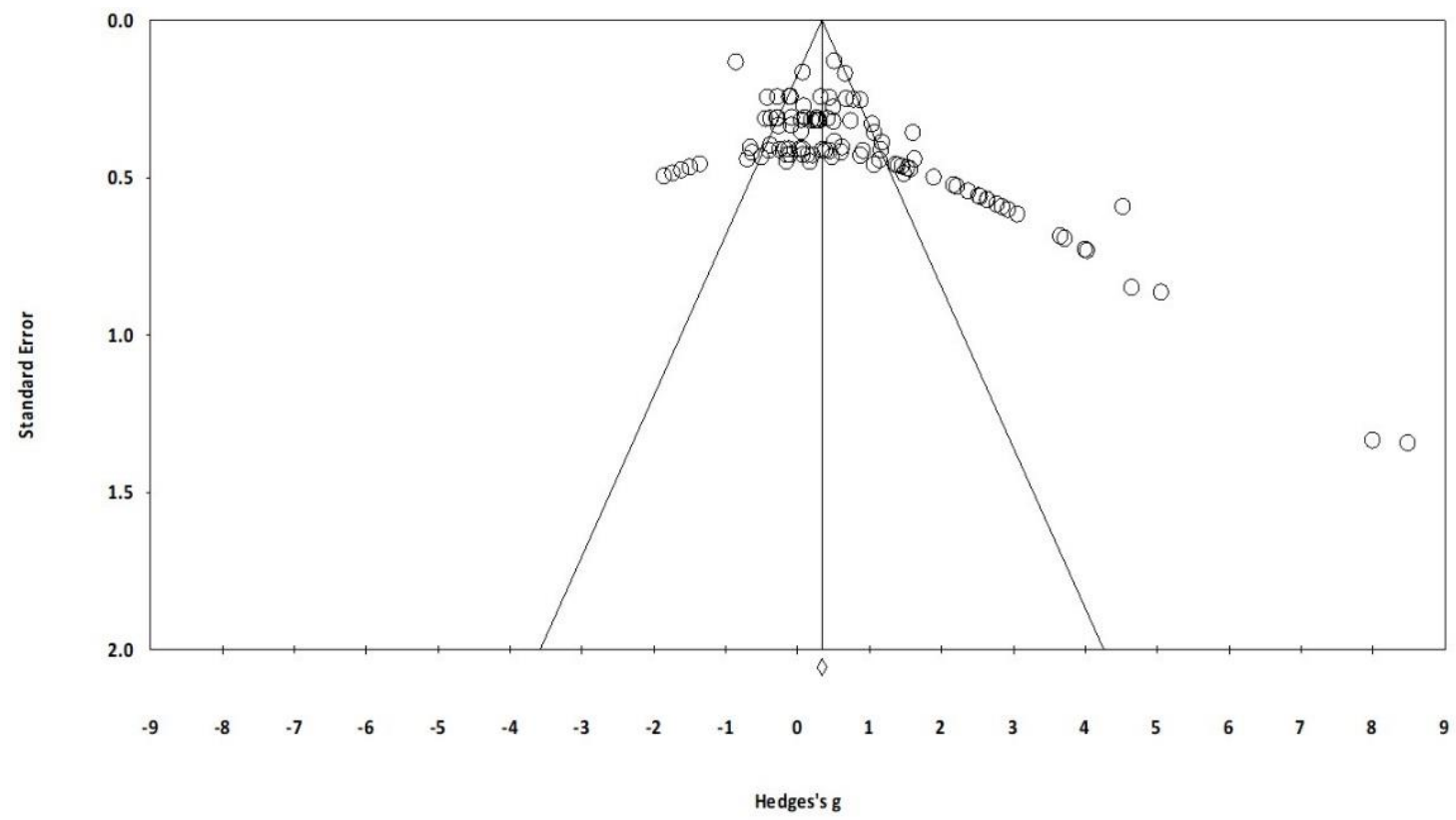

Figure 2. Funnel plot for Hedge's g \& standardized effect for each effect in the meta-analysis. Each of the effect is represented in the plot as a circle. Funnel boundaries represent area where $95 \%$ of the effects are expected to abstain if there were no publication bias. The vertical line represents mean standardized effect of zero. Absence of publication bias is represented when the effects should be equally dispersed on either side of the line. 


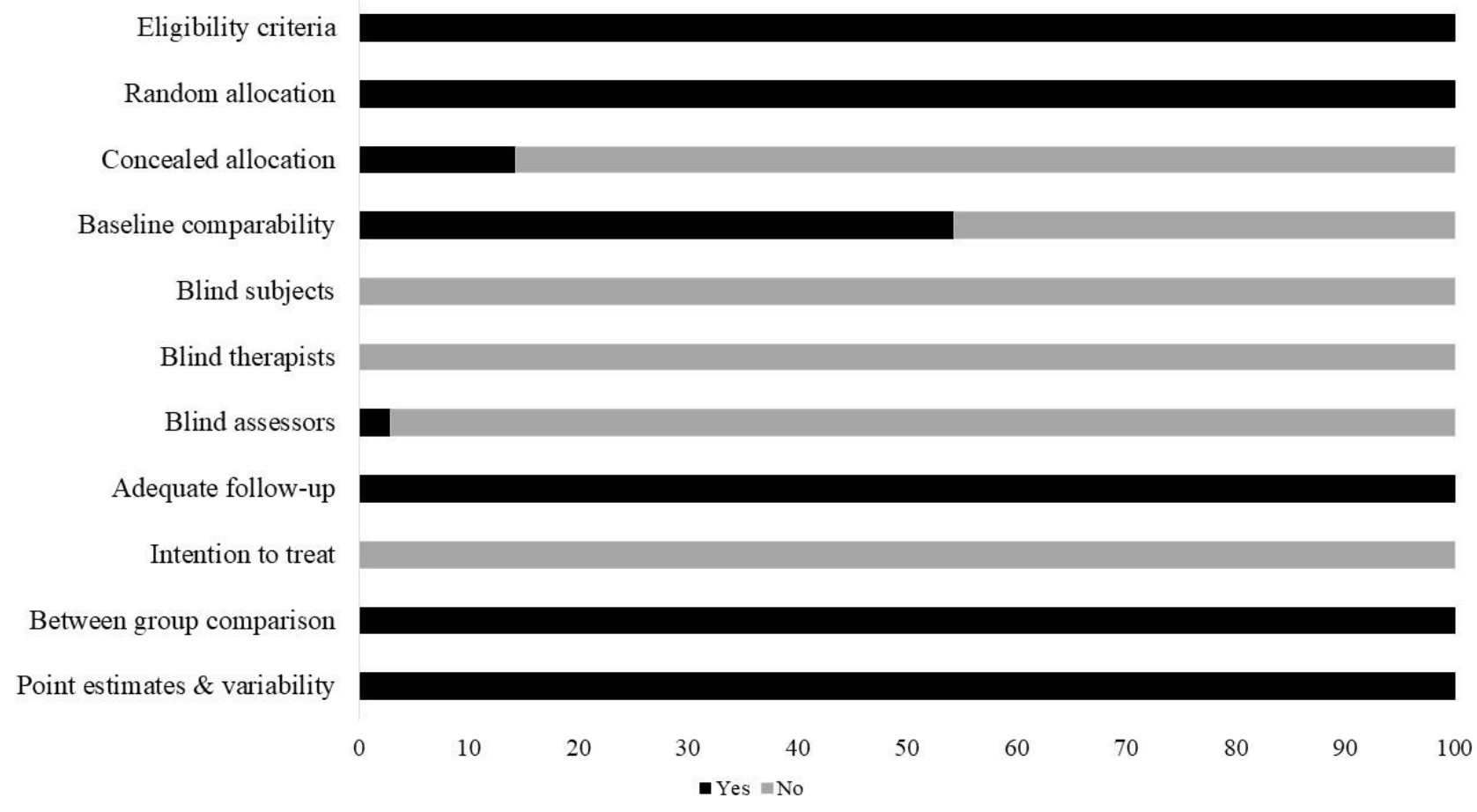

Figure 3. Risk of bias across studies.

\section{Meta-analysis report}

The evaluation of research studies via meta-analysis requires strict inclusion criteria to efficiently limit the heterogeneity [98]. However, among the pooled group of studies post strict inclusion criteria, some amount of unexplained heterogeneity was still observed. Sub-group analysis was then performed for identical studies to evaluate the cause of heterogeneity. The evaluated parameters were the spatio-temporal gait parameters such as, cadence, stride length, gait velocity, coefficient of variability for stride time and stride length. The effects of fast/slow tempo on gait parameters in the included studies was determined by keeping the patient's preferred cadence as reference. Analyses were also conducted to evaluate the effects of dual-task conditions, presence of instructions, and different tempo at which rhythmic auditory cueing was provided on gait parameters. We included a generalized group analysis first combined for all the pooled studies. A separate analysis in addition to clinical controlled trials was performed for high quality randomized controlled trails, for allowing a better interpretation of the direction and magnitude of effects. The main reason for not including the statistical approach within the studies was due to major differences in between assessment methods and lack of descriptive statistics within the manuscript. However, data was not received even after contacting the respective corresponding authors.

\section{Gait velocity}

The meta-analysis on healthy patients revealed (Fig. 4) a large effect size in positive domain with moderate heterogeneity (Hedge's g: $0.85,95 \%$ CI: 0.55 to $1.16, \mathrm{I}^{2}$ : $57.9 \%$, p <0.01). Further, sub-group analysis was performed by dividing the groups in only young/elderly participants.

Young: The analysis for young participants performing gait with rhythmic auditory cueing revealed (Supplementary Fig. 1) beneficial effects with large effect and substantial heterogeneity (g: $0.92,95 \%$ C.I: 0.42 to $\left.1.41, I^{2}: 93.2 \%, p<0.01\right)$. Further, sub-group analysis with non-modulated rhythmic auditory cueing (Supplementary Fig. 2), under a single task condition, revealed a large effect size with substantial heterogeneity (Hedge's g: $1.24,95 \%$ CI: 0.4 to $\left.2, \mathrm{I}^{2}: 90.5 \%, \mathrm{p}<0.01\right)$. The heterogeneity here could be attributed to different interventions utilized by studies. Wellner, et al. [91] for instance, utilized robot assisted gait, and Almeida, et al. [92] analyzed treadmill gait. Moreover, different measures of rhythmic auditory cueing were utilized by 
[99], as the study reported generation of rhythmic patterns by converting the foot strike patterns to rhythmic pattern in real-time.

Further, analysis with fast paced stimuli revealed (Supplementary Fig. 3) large effect size with substantial heterogeneity (g: $1.17,95 \%$ C.I: 0.38 to $1.96, \mathrm{I}^{2}: 91.4 \%$, $\mathrm{p}<0.01)$. Likewise, slow paced stimuli revealed (Supplementary Fig. 4) reduction in gait velocity parameters with medium effect size and substantial heterogeneity (g: $-0.3,95 \%$ C.I: $90.4 \%, \mathrm{I}^{2}: 90.4 \%$, $\mathrm{p}<0.01)$. Here as well, the heterogeneity could be attributed to the type of entrainment used, for instance, low groove, non-motivating cueing and slow cueing were paired together and vice versa for the fast-paced stimuli. These stimuli differ in terms of emotional and expressiveness components, which might be considerably different from each other [68].

Dual task performance with auditory cueing in young participants with/without instructions to walk fast revealed (Supplementary Fig. 5) large effect size with substantial heterogeneity (g: $0.81,95 \%$ C.I: $0.3-1.3, \mathrm{I}^{2}$ : $95.8 \%, \mathrm{p}<0.01)$. Further, performance under pure dualtask conditions without any instructions revealed a medium positive effect size with substantial heterogeneity (g: 0.38 , 95\% C.I: -0.16 to $0.94, \mathrm{I}^{2}: 95 \%$, p<0.01). Here, heterogeneity could be attributed to differential complexities of dual tasks incorporated within the studies, which in published literature have shown to portray different effects on motor performance [8].
Dotov et al. (2017) I 0 Dotov et al. (2017) 0 Dotov et al. (2017) B O Schreiber et al. (2016) Y Hamacher et al. (2016)Y Hamacher et al. (2016) DTY Hamacher et al. (2016) O Hamacher et al. (2016) DTO Yu et al. (2015) Y

Almeida et al. (2015) T Y Sejdic et al. (2012) Y Leow et al. (2014) LG Y Leow et al. (2014) HG Y Leow et al. (2014) Y Franek et al. (2014) SPY Franek et al. (2014) FP Y Franek et al. (2014) Mt Y Franek et al. (2014) NMt Y Lohnes and Earhart (2011) 0 Lohnes and Earhart (2011) FP O Lohnes and Earhart (2011) DT 0 Lohnes and Earhart (2011) DT FP O Lohnes and Earhart (2011) Y Lohnes and Earhart (2011) FP Y Lohnes and Earhart (2011) DT Y Lohnes and Earhart (2011) DT FP Y Arias and Cudeiro (2008) O Wellner et al. (2008) T Y

Baker et al. (2008) SP O Baker et al. (2008) SP INS O Baker et al. (2008) DT SP O Baker et al. (2008) DT SP INS O

Baram and Miller (2007) Y Baker et al. (2007) SP O

Baker et al. (2007) SP INS O Baker et al. (2007) DT SP 0 Baker et al. (2007) DT SP INS O Hausdorff et al. (2007) A.-M. Willems et al. (2006) O Rochester et al. (2005) 0 Rochester et al. (2005) DT McIntosh et al. (1997) O
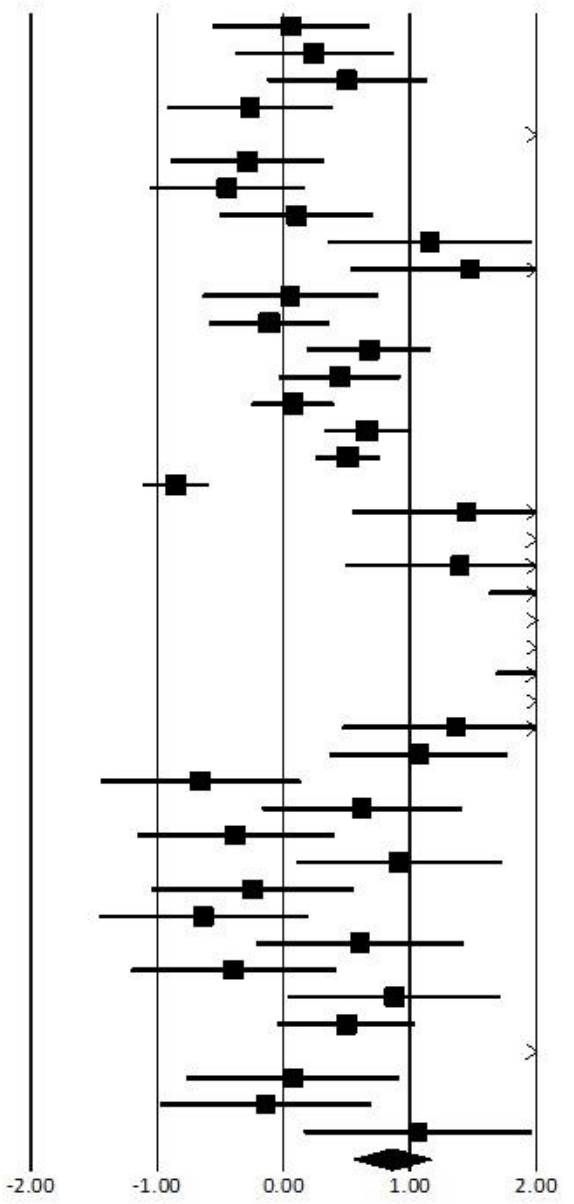

Figure 4. Forest plot illustrating individual studies evaluating the effects of rhythmic auditory cueing on gait velocity among healthy young and elderly participants. A negative effect size indicated reduction in gait velocity; a positive effect size indicated enhancement in gait velocity. Weighted effect sizes; Hedge's g (boxes) and 95\% C.I (whiskers) are presented, demonstrating repositioning errors for individual studies. The (Diamond) represents pooled effect sizes and 95\% CI. A negative mean difference indicates a favorable outcome for control groups; a positive mean difference indicates a favorable outcome for experimental groups. (O: Old, Y: Young, FP: Fast paced, SP: Slow paced, DT: Dual-task, I: Isosynchronous, B: Biological variability, LG: Low groove, HG: High groove, INS: Instructions, Mt: Motivating feedback, NMt: Non-motivating feedback). 


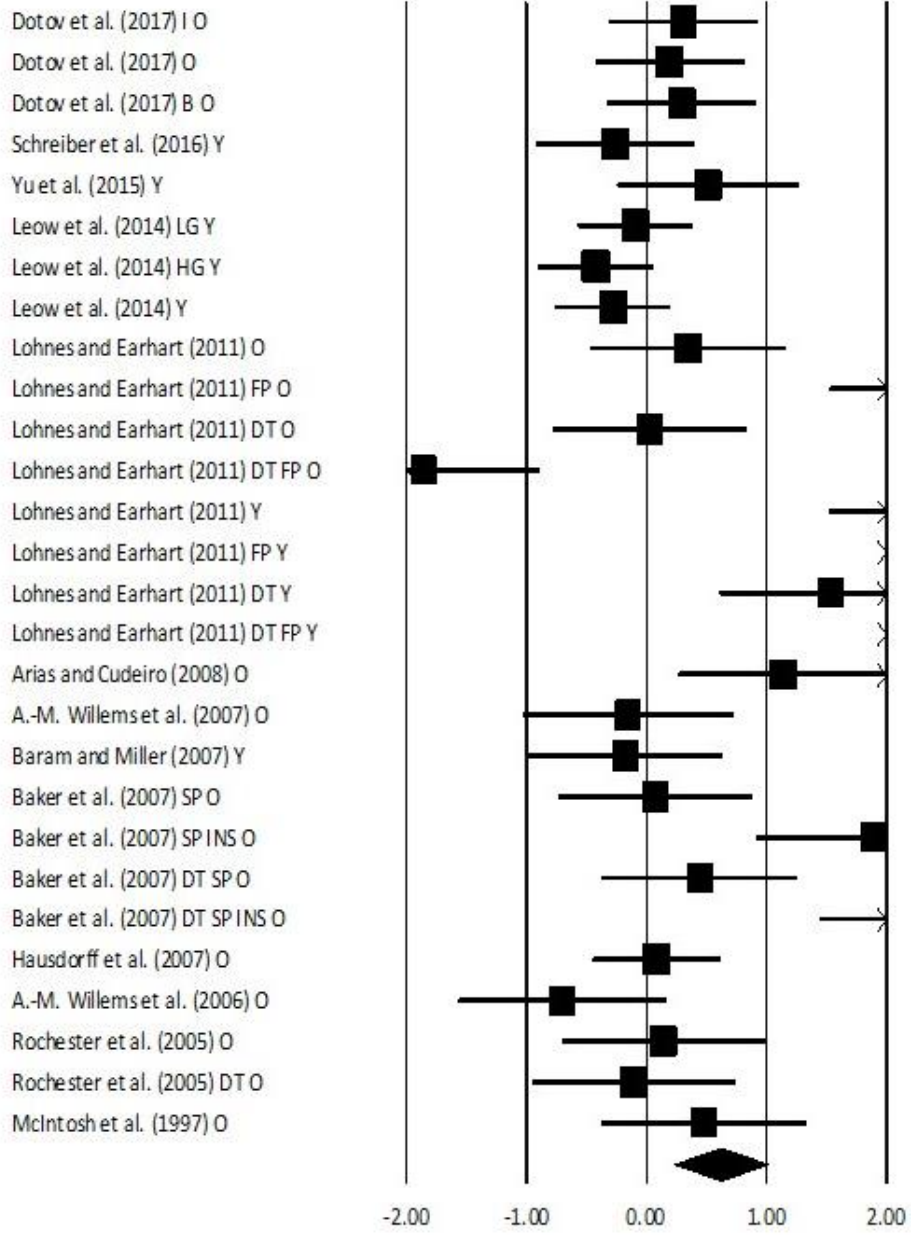

Figure 5. Forest plot illustrating individual studies evaluating the effects of rhythmic auditory cueing on stride length among healthy young and elderly participants. A negative effect size indicated reduction in stride length; a positive effect size indicated enhancement in stride length. Weighted effect sizes; Hedge's g (boxes) and 95\% C.I (whiskers) are presented, demonstrating repositioning errors for individual studies. The (Diamond) represents pooled effect sizes and 95\% CI. A negative mean difference indicates a favorable outcome for control groups; a positive mean difference indicates a favorable outcome for experimental groups. (O: Old, Y: Young, FP: Fast paced, SP: Slow paced, DT: Dual-task, I: Isosynchronous, B: Biological variability, LG: Low groove, HG: High groove, INS: Instructions, Mt: Motivating feedback, NMt: Nonmotivating feedback).
Old: The analysis for old participants performing gait with rhythmic auditory cueing revealed (Supplementary Fig. 6) beneficial effects with medium effect and substantial heterogeneity (g: $0.68,95 \%$ C.I: 0.28 to $\left.1, \mathrm{I}^{2}: 81 \%, \mathrm{p}<0.01\right)$. Further, sub-group analysis with non-modulated rhythmic auditory cueing revealed (Supplementary Fig. 7), under a single task condition, revealed a medium effect size with substantial heterogeneity (Hedge's g: $0.73,95 \%$ CI: 0.2 to $1.2, \mathrm{I}^{2}$ : $80.2 \%, \mathrm{p}<0.01)$. Here, Dotov, Bayard, de Cock, Geny, Driss, Garrigue, Bardy and Dalla Bella [100] evaluated the effectiveness of feedbacks which were isosynchronous, and with/without biological variability. Possibly, the heterogeneity in the sub-group analysis could be attributed to the differential cueing utilized. Further, only one study analyzed the effects of fast paced stimuli amongst elderly and further couldn't be included in sub-group analysis [67]. Slow paced stimuli, with/without verbal instructions revealed (Supplementary Fig. 8) enhancements in gait velocity parameters with small effect size and negligible heterogeneity (g: 0.25 ,
95\% C.I: -0.49 to $\left.1, \mathrm{I}^{2}: 0 \%, \mathrm{p}>0.05\right)$. Additional, subgroup analysis revealed a considerable effect of verbal instructions over gait velocity i.e. analysis for performance without verbal instructions revealed a negative medium effect size with negligible heterogeneity (g: $-0.4,95 \%$ C.I: -0.98 to $0.18, \mathrm{I}^{2}: 0 \%, \mathrm{p}>0.05$ ), and including verbal instructions revealed a positive large effect size with negligible heterogeneity (g: 0.92, 95\% C.I: 0.32 to $\left.1.5, \mathrm{I}^{2}: 0 \%, \mathrm{p}>0.05\right)$. Dual task performance with auditory cueing in elderly participants with/without instructions to walk fast revealed (Supplementary Fig. 9) a medium positive effect size with substantial heterogeneity (g: $0.58,95 \%$ C.I: -0.05 to $1.2, \mathrm{I}^{2}: 79.2 \%$, $\mathrm{p}>0.05)$. Performing under non-modulated rhythmic auditory cueing without any instructions with dual task revealed (Supplementary Fig. 10) a medium positive effect size (g: $0.43,95 \%$ C.I: -0.44 to $1.3, \mathrm{I}^{2}$ : $13.4 \%$, $\mathrm{p}>0.05$ ) with negligible heterogeneity.

\section{Stride length}


The meta-analysis on healthy patients revealed (Fig. 5) a medium effect size in positive domain with substantial heterogeneity (Hedge's g: $0.61,95 \% \mathrm{CI}: 0.23$ to $1, \mathrm{I}^{2}$ : $58.8 \%, \mathrm{p}<0.05)$. Further, sub-group analysis was performed by dividing the groups in only young/elderly participants.

Young: The analysis for young participants performing gait with rhythmic auditory cueing revealed (Supplementary Fig. 11) beneficial effects with large effect and substantial heterogeneity (g: 1.2, 95\% C.I: 0.38 to $\left.2.85, \mathrm{I}^{2}: 92 \%, \mathrm{p}<0.01\right)$. Further, sub-group analysis with non-modulated rhythmic auditory cueing revealed (Supplementary Fig. 12), under a single task condition, revealed a large effect size with substantial heterogeneity (Hedge's g: 0.81, 95\% CI: -0.5 to $1.7, \mathrm{I}^{2}: 88 \%$, p<0.01). Further, analysis with fast paced stimuli revealed small effect size with substantial heterogeneity (g: $-0.01,95 \%$ C.I: -0.4 to $0.4, \mathrm{I}^{2}: 92.5 \%, \mathrm{p}<0.01$ ). The heterogeneity as stated before could be attributed to differential rhythmic stimuli utilized by studies. Moreover, none of the studies analyzing a slow-paced stimulus evaluated stride length. Dual task performance was analyzed in only one included study. Therefore, no further analysis could be carried out to evaluate the effects of higher information processing constraints on stride length.

Dotov et al. (2017) IO
Dotov et al. (2017) O
Dotov et al. (2017) B O
Schreiber et al. (2016) Y
Yu et al. (2015) Y
Lohnes and Earhart (2011) O
Lohnes and Earhart (2011) FP O
Lohnes and Earhart (2011) DT O
Lohnes and Earhart (2011) DT FP O
Lohnes and Earhart (2011) Y
Lohnes and Earhart (2011) FP Y
Lohnes and Earhart (2011) DT Y
Lohnes and Earhart (2011) DT FP Y
Arias and Cudeiro (2008) O
Baker et al. (2007) SP O
Baker et al. (2007) SP INS O
Baker et al. (2007) DT SP O
Baker et al. (2007) DT SP INS O
A.-M. Willems et al. (2006) O
Rochester et al. (2005) O
Rochester et al. (2005) DT O
Mclntosh et al. (1997) O

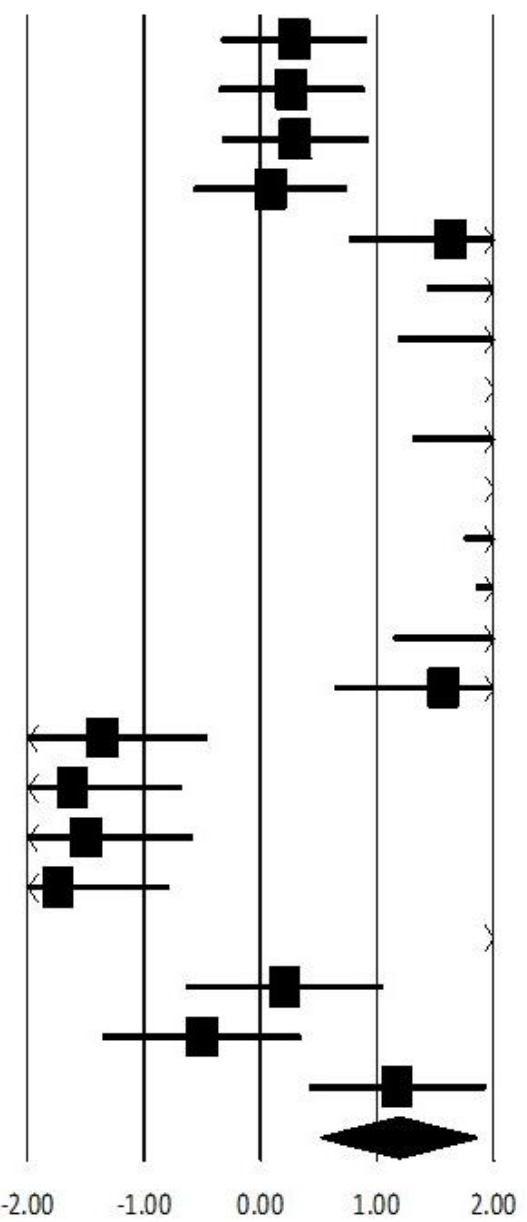

Figure 6. Forest plot illustrating individual studies evaluating the effects of rhythmic auditory cueing on cadence among healthy young and elderly participants. A negative effect size indicated reduction in step frequency; a positive effect size indicated enhancement in step frequency. Weighted effect sizes; Hedge's $\mathrm{g}$ (boxes) and 95\% C.I (whiskers) are presented, demonstrating repositioning errors for individual studies. The (Diamond) represents pooled effect sizes and 95\% CI. A negative mean difference indicates a favorable outcome for control groups; a positive mean difference indicates a favorable outcome for experimental groups. (O: Old, Y: Young, FP: Fast paced, SP: Slow paced, DT: Dual-task, I: Isosynchronous, B: Biological variability, LG: Low groove, HG: High groove, INS: Instructions, Mt: Motivating feedback, NMt: Nonmotivating feedback)
Old: The analysis for old participants performing gait with rhythmic auditory cueing revealed (Supplementary Fig. 13) beneficial effects with medium effect and substantial heterogeneity (g: 0.39 , 95\% C.I: 0.01 to $\left.0.78, \mathrm{I}^{2}: 77 \%, \mathrm{p}<0.01\right)$. Further, sub-group analysis with non-modulated rhythmic auditory cueing revealed
(Supplementary Fig. 14), under a single task condition, revealed a small effect size with negligible heterogeneity (Hedge's g: $0.22,95 \%$ CI: -0.03 to $0.46, \mathrm{I}^{2}: 10.5 \%$, $\mathrm{p}>0.05)$. Further, one study each analyzed the effects of fast, slow paced stimuli amongst elderly and further couldn't be included in sub-group analysis $[67,101]$. Dual 
task performance with auditory cueing in elderly participants was analyzed amongst two studies [67, 94], a small effect size with negligible heterogeneity (g: -0.03 , 95\% C.I: -0.64 to $0.56, \mathrm{I}^{2}: 0 \%, \mathrm{p}>0.05$ ).

\section{Cadence}

The meta-analysis on healthy patients revealed (Fig. 6) a large effect size in positive domain with moderate heterogeneity (Hedge's g: $1.2,95 \% \mathrm{CI}: 0.51$ to $1.8, \mathrm{I}^{2}$ : $41.9 \%, \mathrm{p}<0.01)$. Further, sub-group analysis was performed by dividing the groups in only young/elderly participants.

Young: Further, sub-group analysis with nonmodulated rhythmic auditory cueing revealed (Supplementary Fig. 15), under a single task condition, revealed a large effect size with substantial heterogeneity (Hedge's g: $1.76,95 \%$ CI: -0.29 to $3.8, \mathrm{I}^{2}: 93.2 \%$, p <0.01). Only one study performed [67], rhythmic auditory cueing with fast pace and no study analyzed the effects with slow paced stimulus. Therefore, no additional analysis was carried out. Dual task performance was analyzed in only one included study. Therefore, no further analysis could be carried out to evaluate the effects of higher information processing constraints on cadence.

Old: The analysis for old participants performing gait with rhythmic auditory cueing revealed (Supplementary Fig. 16) beneficial effects with medium effect and substantial heterogeneity (g: $0.78,95 \%$ C.I: 0.01 to $\left.1.54, \mathrm{I}^{2}: 91.5 \%, \mathrm{p}<0.01\right)$. Sub-group analysis with non-modulated rhythmic auditory (Supplementary Fig. 17), under a single task condition, revealed a large effect size with substantial heterogeneity (Hedge's g: 1.02, 95\% CI: 0.19 to $\left.1.84, \mathrm{I}^{2}: 88.6 \%, \mathrm{p}<0.01\right)$. Further, one study each analyzed the effects of fast, slow paced stimuli amongst elderly and further couldn't be included in subgroup analysis [67, 101]. Dual task performance with auditory cueing in elderly participants was analyzed amongst two studies [67, 94], a medium effect size with substantial heterogeneity (g: 0.68, 95\% C.I: -0.03 to -1.41 , $\left.I^{2}: 96 \%, \mathrm{p}<0.01\right)$.

\section{Coefficient of variability stride time}

Analysis of coefficient of variability for stride time revealed (Supplementary Fig. 18) a small effect in positive domain with substantial heterogeneity (g: 0.21 , 95\% C.I: -0.42 to $0.85, \mathrm{I}^{2}: 67.7 \%$, p<0.05). Further, in a sub-group analysis for only old participants revealed a medium effect size in positive domain with substantial heterogeneity (g: $0.4,95 \%$ C.I: -0.33 to $1.13, \mathrm{I}^{2}$ : $63 \%$, $\mathrm{p}<0.05)$ [102-104].

\section{Coefficient of variability stride length}

Analysis of coefficient of variability for stride length revealed (Supplementary Fig. 19) a medium effect in positive domain with moderate heterogeneity $(\mathrm{g}: 0.76$, 95\% C.I: 0.43 to $1.1, \mathrm{I}^{2}: 48.7 \%$, p>0.05) [102, 104, 105]. Further, in a sub-group analysis for only young participants with non-modulated rhythmic auditory cueing revealed a medium effect size in positive domain with negligible heterogeneity (g: $0.47,95 \%$ C.I: -0.09 to $\left.0.85, \mathrm{I}^{2}: 4.7 \%, \mathrm{p}>0.05\right)[104,105]$. Likewise, for only old participants a large effect size in positive domain with negligible heterogeneity ( $\mathrm{g}: 1.01,95 \%$ C.I: -0.17 to 2.2 , $\left.I^{2}: 0 \%, p>0.05\right)$ was observed $[102,104]$.

\section{DISCUSSION}

The primary objective of this present systematic review and meta-analysis was to synthesize the current state of knowledge for effects that rhythmic auditory cueing might lay over aging gait. Out of thirty-four included studies, $88 \%$ studies reported beneficial effects of rhythmic auditory cueing on primary spatiotemporal gait parameters.

Typically, spatiotemporal parameters of gait worsen with age $[19,106]$. Callisaya, Beare, Phan, Blizzard, Thrift, Chen and Srikanth [107], studied age associated decline in brain structure with gait performance, and linked a reduction in gait velocity, stride length, cadence with white matter atrophy, lesions, hippocampal atrophy, and gray matter atrophy with cerebral infarcts, respectively [107, 108]. Moreover, research suggests that degenerative changes in the fronto-striatal circuits might add increasing bi-directional stress on automated control for posture, gait and cognitive processing [109-111]. Possibly, explaining the loss of gait rhythmicity in elderly (see also, Nombela, et al. [56]). Likewise, increased energy expenditure [108], weak musculoskeletal structure associated variability in muscle contraction, and force production add towards the woes [112]. The current metaanalysis reported enhancements in gait velocity (g: 0.68), stride length (0.39) and cadence (0.78), post application of rhythmic auditory cueing in elderly population groups. Likewise, beneficial effects of rhythmic auditory cueing were also observed in gait amongst younger population groups.

Several mechanisms have been suggested to ascertain the beneficial effects of rhythmic auditory cueing. Rizzo, Raghavan, McCrery, Oh-Park and Verghese [113] for instance, speculated that auditory entrainment while performing gait might act as an efficient distractor. In addition, the auditory entrainment might also have aided in reducing the errors while executing the gait $[114,115]$. Possibly, by acting as an external guidance for "heel- 
contact" and "push-off" timings. Moreover, application of auditory entrainment is believed to allow enhancement in gait performance by bypassing or facilitating the degenerated basal ganglia-motor loop via alternative pathways [116-118]. Cunnington, Iansek, Bradshaw and Phillips [119] suggested that the external stimulation by entrainment might surpass deficient pallidal-cortical projections, and can directly serve an input supplementary motor area, thereby reducing the onset of motor deficit and aiding in performance. Moreover, the external cueing has shown to allow modulation of neuromagnetic $\beta$ oscillations in auditory cortex, cerebellum, inferior frontal gyrus, somatosensory area and sensorimotor cortex [120], and reduce hemispheric asymmetry [121]. Neuroimaging studies reveal enhance activation in inferior colliculi [122], cerebellum, brainstem [117, 123], sensorimotor cortex [124, 125], further instigating cortico-cerebellar network re-organization [126]. Another crucial factor that considerably influences the aging gait is "change in tempo". Neurophysiological analysis suggests, increased neuronal activation in fronto-occipital networks [127], and excitability of the spinal motor neurons by reticulospinal pathways, with fast-paced entrainment. A paced-stimuli is thought to reduce the response time, limit the stagnating effects of constant entrainment over fractal scaling of stride times from healthy $1 / \mathrm{f}$ structure [128130], and optimizing the velocity and acceleration profiles of joint motions by scaling movement time [59].

The present-meta-analysis also observed enhancements in the spatiotemporal parameters while performing dual-tasks, for both age groups. According to literature, dual-task performance predisposes to gait instability and falls by increasing cognitive motor interferences, across age groups [8, 131-133]. Interpretations from our results suggest that rhythmic auditory cueing counteracts cognitive constraints imposed by cognitively demanding dual-tasks such as carrying a tray and that this cueing might be useful in counteracting fall while carrying out activities of daily living [8]. Lohnes and Earhart [67], suggested that co-performance of dual-tasks with rhythmic auditory cueing might allow enhancements (or even stability) in performance, by possibly freeing up cognitive resources for dual-task performance. The authors also mentioned the influence of task complexity across age groups. Possibly, the freed up cognitive resources might not be sufficient especially in elderly to perform complex dual-tasks, such as coin transfer [134], and sentence reciting tasks [135]. This might possibly explain the reduced dual tasks costs on gait performance in young participants. In addition, the enhanced performance could also be attributed as to how the participants might perceive the auditory entrainment based on their cognitive capabilities. Wittwer, Webster and Hill [136], and Thaut, Miltner, Lange, Hurt and
Hoemberg [137], suggested a strong relationship in between the cognitive capabilities and the ability to interpret and discern the structure of a beat. Thereby, suggesting a better rhythmic perception and interpretation by younger population groups as compared to their older counterparts.

Moreover, the progressive degradation of neuromuscular structures with aging has further been suggested to alleviate the threshold for action relevant acoustic input [138]. To counteract this deficiency use of ecologically valid acoustic feedback has been suggested [138]. The ecologically valid action related sounds might enhance saliency of sensory information concerning spatiotemporal information, thereby aiding in movement execution [100, 138-141]. This was also demonstrated by Dotov, et al. [100], here the authors demonstrated beneficial effects in parkinsonian and healthy gait parameters with biologically variable rhythmic auditory cueing as compared to isosynchronous cueing. Moreover, recent research has also revealed the possibilities of including emotional [113], motivational [68], and expressiveness [142], component in auditory entrainment to portray differential effects on gait parameters. Unfortunately, lack of pertinent, repeatable literature concerning the specific type of modified auditory feedback makes it difficult to interpret, as to which type of feedback might be most optimal, and for which age groups. We suggest future studies to replicate data concerning the use of ecological auditory entrainment across different age groups, to allow a reliable interpretation, which could then be included in gait rehabilitation protocols. Moreover, we also suggest future researchers to analyze the "entrainment effects" while multitasking in high-stress situations pertinent to modern day scenarios (for example, walking and texting, listening to music while crossing a traffic light).

This current meta-analysis also reported an increase in coefficient of stride-time and length variability in elderly participants with rhythmic auditory cueing. Based, on the published literature initial increase in variability during learning paradigm is efficient for improving gait performance [143]. Here, interpretations could possibly be drawn from "dynamic system theory" [144]. The theory suggests that a biological system might allow variability to identify and self-organize the most stable and viable outcome [144, 145]. Thereby, interpretations could be made for regulating gait amongst young and elderly population groups to regulate gait when passing through fall-prone environments [41]. The present metaanalysis did not evaluate the the influence of gait training with rhythmic auditory cueing on ageing gait. Whereas, training regimes with auditory entrainment have demonstrated reduced variability in parkinsonism [101, 146], and stroke [126]. We suggest future research to 
address this gap in the literature and evaluate the effects of long term training with rhythmic auditory cueing on aging gait.

Finally, we believe that the benefits of auditory entrainment might surpass that of co-treatment techniques (for instance, biofeedback, virtual reality, physiotherapy etc.) because of its economical nature, and high viability [77, 78]. The rhythmic entrainment factor could be utilized with music in rehabilitation, day to day lives. This could allow benefits in both psycho-physiological domains [147-151]. For instance, improving stress, mediating arousal, emotions, internal motivation, memory, attention, executive functions [152], power [153], and endurance [154]. Moreover, it is important to consider that the retention of enhancements in gait parameters relies not only on the training received in the clinic but also depends largely on how much the patient follows the treatment protocol at home. Lim, et al. [13] for instance, reported enhancement in parkinsonian gait activity to 35 minutes per day (qualifying the 30 minutes criteria by WHO [155]). We believe that delivering this type of home-based intervention could possibly be beneficial for people lacking proper exposure to medical interventions in developing countries [156]. For instance, a booming number of smartphone devices in developing countries [157], can be used as a delivery tool while using a simple metronome app such as, Walkmate [129], or Listenmee [158], which with proper medical guidance might allow curbing the motor deficits associated with aging [159]. We also suggest the use of rhythmic auditory cueing as an adjunct to other rehabilitation strategies, for instance, dance, tai-chi, aerobics, as it might enhance the rehabilitation progress by focusing on both psychophysiological components.

To the best of our knowledge, this present review for the first time analyzed the effects of auditory entrainment on aging gait. The present findings are in agreement with systematic reviews and meta-analysis carried out to analyze auditory entrainment effect on stroke [66], cerebral palsy [160], and parkinsonism [57, 161]. In conclusion, this review strongly suggests the incorporation of rhythmic auditory cueing for enhancing gait performance with aging gait. The results from the meta-analysis also direct towards the possible use of auditory entrainment to reduce the incidence of falls in high-stress situations.

\section{Supplemental data}

Supplemental data are available online at www.aginganddisease.org/EN/10.14336/AD.2017.1031.

\section{Competing Financial Interests}

No financial interests are declared.

\section{Acknowledgement}

The publication of this article was funded by the Open Access fund of Leibniz Universität Hannover.

\section{Supplemental data}

Supplemental data are available online.

\section{References}

[1] Tinetti ME, Speechley M, Ginter SF (1988). Risk factors for falls among elderly persons living in the community. N Engl J Med, 319: 1701-1707

[2] Boudarham J, Roche N, Pradon D, Bonnyaud C, Bensmail D, Zory R (2013). Variations in Kinematics during Clinical Gait Analysis in Stroke Patients. PLoS ONE, 8: e66421

[3] Zecevic AA, Salmoni AW, Speechley M, Vandervoort AA (2006). Defining a fall and reasons for falling: comparisons among the views of seniors, health care providers, and the research literature. Gerontologist, 46: 367-376

[4] Ageing WHO, Unit LC (2008) WHO global report on falls prevention in older age, World Health Organization

[5] Segev-Jacubovski O, Herman T, Yogev-Seligmann G, Mirelman A, Giladi N, Hausdorff JM (2011). The interplay between gait, falls and cognition: can cognitive therapy reduce fall risk? Expert Rev Neurother, 11: 1057-1075

[6] Jahn K, Zwergal A, Schniepp R (2010). Gait Disturbances in Old Age: Classification, Diagnosis, and Treatment From a Neurological Perspective. Dtsch Arztebl Int, 107: 306-316

[7] de Moraes Barros GDV Falls in elderly people. Lancet, 367: 729-730

[8] Ghai S, Ghai I, Effenberg AO (2017). Effects of dual tasks and dual-task training on postural stability: a systematic review and meta-analysis. Clin Interv Aging, 12: 557-577

[9] Cromwell RL, Newton RA (2004). Relationship between balance and gait stability in healthy older adults. J Aging Phys Act, 12: 90-100

[10] Talbot LA, Musiol RJ, Witham EK, Metter EJ (2005). Falls in young, middle-aged and older community dwelling adults: perceived cause, environmental factors and injury. BMC Public Health, 5: 86-86

[11] Salzman B (2010). Gait and balance disorders in older adults. Am Fam Physician, 82: 61-68

[12] Herman T, Giladi N, Gruendlinger L, Hausdorff JM (2007). Six weeks of intensive treadmill training improves gait and quality of life in patients with Parkinson's disease: a pilot study. Arch Phys Med Rehabil, 88: 1154-1158

[13] Lim I, van Wegen E, Jones D, Rochester L, Nieuwboer A, Willems A-M, et al. (2010). Does cueing training 
improve physical activity in patients with Parkinson's disease? Neurorehabil Neural Repair, 24: 469-477

[14] Bhatt T, Espy D, Yang F, Pai Y-C (2011). Dynamic gait stability, clinical correlates, and prognosis of falls among community-dwelling older adults. Arch Phys Med Rehabil, 92: 799-805

[15] Stevens JA, Corso PS, Finkelstein EA, Miller TR (2006). The costs of fatal and non-fatal falls among older adults. Inj Prev, 12: 290-295

[16] Niino N, Tsuzuku S, Ando F, Shimokata H (2000). Frequencies and circumstances of falls in the National Institute for Longevity Sciences, Longitudinal Study of Aging (NILS-LSA). J Epidemiol, 10: S90-94

[17] Kenny R, Rubenstein LZ, Tinetti ME, Brewer K, Cameron KA, Capezuti L, et al. (2011). Summary of the updated American Geriatrics Society/British Geriatrics Society clinical practice guideline for prevention of falls in older persons. J Am Geriatr Soc, 59: 148-157

[18] Tinetti ME, Kumar C (2010). The patient who falls:"It's always a trade-off'. Jama, 303: 258-266

[19] Thaler-Kall K, Peters A, Thorand B, Grill E, Autenrieth CS, Horsch A, et al. (2015). Description of spatiotemporal gait parameters in elderly people and their association with history of falls: results of the population-based cross-sectional KORA-Age study. BMC Geriatrics, 15: 32

[20] Schmitz A, Silder A, Heiderscheit B, Mahoney J, Thelen DG (2009). Differences in lower-extremity muscular activation during walking between healthy older and young adults. J Electromyogr Kinesiol, 19: 1085-1091

[21] Hamacher D, Singh NB, Van Dieen JH, Heller MO, Taylor WR (2011). Kinematic measures for assessing gait stability in elderly individuals: a systematic review. J R Soc Interface, 8: 1682-1698

[22] Callisaya M, Blizzard L, McGinley JL, Srikanth V (2012). Risk of falls in older people during fastwalking-the TASCOG study. Gait Posture, 36: 510-515

[23] Reelick MF, van Iersel MB, Kessels RP, Rikkert MGO (2009). The influence of fear of falling on gait and balance in older people. Age Ageing, 38: 435-440

[24] DeVita P, Hortobagyi T (2000). Age causes a redistribution of joint torques and powers during gait. $\mathrm{J}$ Appl Physiol, 88: 1804-1811

[25] de Melker Worms JLA, Stins JF, van Wegen EEH, Loram ID, Beek PJ (2017). Influence of focus of attention, reinvestment and fall history on elderly gait stability. Physiol Rep, 5: e13061

[26] Medeiros HBdO, Araújo DSMSd, Araújo CGSd (2013). Age-related mobility loss is joint-specific: an analysis from 6,000 Flexitest results. Age, 35: 23992407

[27] Masters RSW, Maxwell J (2008). The theory of reinvestment. Int Rev Sport Exer Psychol, 1: 160-183

[28] Masters RSW (1992). Knowledge, knerves and knowhow: The role of explicit versus implicit knowledge in the breakdown of a complex motor skill under pressure. Brit J Psychol, 83: 343-358
Kurlan R (2005). "Fear of falling" gait: a potentially reversible psychogenic gait disorder. Cogn Behav Neurol, 18: 171-172

[30] Tinetti ME, Richman D, Powell L (1990). Falls efficacy as a measure of fear of falling. J Gerontol, 45: P239P243

[31] Cromwell RL, Newton RA, Forrest G (2002). Influence of vision on head stabilization strategies in older adults during walking. J Gerontol A Biol Sci Med Sci, 57: M442-M448

[32] Giladi N, Herman T, Reider G, II, Gurevich T, Hausdorff JM (2005). Clinical characteristics of elderly patients with a cautious gait of unknown origin. $\mathbf{J}$ Neurol, 252: 300-306

[33] Young WR, Mark Williams A (2015). How fear of falling can increase fall-risk in older adults: Applying psychological theory to practical observations. Gait Posture, 41: 7-12

[34] Young WR, Olonilua M, Masters RS, Dimitriadis S, Williams AM (2016). Examining links between anxiety, reinvestment and walking when talking by older adults during adaptive gait. Exp Brain Res, 234: 161-172

[35] Spreng RN, Wojtowicz M, Grady CL (2010). Reliable differences in brain activity between young and old adults: a quantitative meta-analysis across multiple cognitive domains. Neurosci Biobehav Rev, 34: 11781194

[36] Ghai S, Driller M, Ghai I (2017). Effects of joint stabilizers on proprioception and stability: A systematic review and meta-analysis. Phys Ther Sport, 25: 65-75

[37] Ghai S, Driller MW, Masters RSW (2016). The influence of below-knee compression garments on knee-joint proprioception. Gait Posture, [Epub ahead of print]

[38] Ghai S (2016) Proprioception and Performance: The role of below-knee compression garments and secondary tasks. University of Waikato, Hamilton, New Zealand

[39] Lee JH, Chun MH, Jang DH, Ahn JS, Yoo JY (2007). A comparison of young and old using threedimensional motion analyses of gait, sit-to-stand and upper extremity performance. Aging Clin Exp Res, 19: 451-456

[40] Mertz KJ, Lee D-c, Sui X, Powell KE, Blair SN (2010). Falls Among Adults: The Association of Cardiorespiratory Fitness and Physical Activity with Walking-Related Falls. Am J Prev Med, 39: 15-24

[41] Schabrun SM, van den Hoorn W, Moorcroft A, Greenland C, Hodges PW (2014). Texting and Walking: Strategies for Postural Control and Implications for Safety. PLoS ONE, 9: e84312

[42] Demura S, Uchiyama M (2009). Influence of cell phone email use on characteristics of gait. Eur J Sport Sci, 9: 303-309

[43] Lin M-IB, Lin K-H (2016). Walking while Performing Working Memory Tasks Changes the Prefrontal Cortex Hemodynamic Activations and Gait Kinematics. Front Behav Neurosci, 10: 92 
[44] Nagata T, Uno H, Perry MJ (2010). Clinical consequences of road traffic injuries among the elderly in Japan. BMC Public Health, 10: 375

[45] de Rooij IJ, van de Port IG, Meijer JG (2016). Effect of Virtual Reality Training on Balance and Gait Ability in Patients With Stroke: Systematic Review and MetaAnalysis. Phys Ther, 96: 1905-1918

[46] Pizzolato C, Reggiani M, Saxby DJ, Ceseracciu E, Modenese L, Lloyd DG (2017). Biofeedback for Gait Retraining Based on Real-Time Estimation of Tibiofemoral Joint Contact Forces. IEEE Trans Neural Syst Rehabil Eng, 25: 1612-1621

[47] Eng JJ, Tang PF (2007). Gait training strategies to optimize walking ability in people with stroke: A synthesis of the evidence. Expert Rev Neurother, 7: 1417-1436

[48] Bastian A, Keller JL (2014). A Home Balance Exercise Program Improves Walking in People with Cerebellar Ataxia. Neurorehabil Neural Repair, 28: 770-778

[49] Hackney ME, Earhart GM (2009). Effects of Dance on Movement Control in Parkinson's Disease: A Comparison of Argentine Tango and American Ballroom. J Rehabil Med, 41: 475-481

[50] Mehrholz J, Kugler J, Storch A, Pohl M, Elsner B, Hirsch K (2015). Treadmill training for patients with Parkinson's disease. Cochrane Database Syst Rev: Cd007830

[51] Thaut MH, Abiru M (2010). Rhythmic auditory stimulation in rehabilitation of movement disorders: a review of current research. Music Percept, 27: 263-269

[52] Thaut MH, McIntosh GC, Hoemberg V (2014). Neurobiological foundations of neurologic music therapy: rhythmic entrainment and the motor system. Front Psychol, 5: 1185

[53] Low S, Ang LW, Goh KS, Chew SK (2009). A systematic review of the effectiveness of Tai Chi on fall reduction among the elderly. Arch Gerontol Geriatr, 48: 325-331

[54] Huang Z-G, Feng Y-H, Li Y-H, Lv C-S (2017). Systematic review and meta-analysis: Tai Chi for preventing falls in older adults. BMJ Open, 7: e013661

[55] Rubenstein LZ, Stevens JA, Scott V (2008) Interventions to prevent falls among older adults. In Handbook of injury and violence prevention pp. 37-53, Springer

[56] Nombela C, Hughes LE, Owen AM, Grahn JA (2013). Into the groove: can rhythm influence Parkinson's disease? Neurosci Biobehav Rev, 37: 2564-2570

[57] Spaulding SJ, Barber B, Colby M, Cormack B, Mick T, Jenkins ME (2013). Cueing and gait improvement among people with Parkinson's disease: a metaanalysis. Arch Phys Med Rehabil, 94: 562-570

[58] Lim I, van Wegen E, de Goede C, Deutekom M, Nieuwboer A, Willems A, et al. (2005). Effects of external rhythmical cueing on gait in patients with Parkinson's disease: a systematic review. Clin Rehabil, 19: 695-713

[59] Thaut MH (2005) Rhythm, music, and the brain: Scientific foundations and clinical applications Vol. 7, Routledge
[60] Raglio A (2015). Music therapy interventions in Parkinson's disease: the state-of-the-art. Front Neurol, 6

[61] Shelton J, Kumar GP (2010). Comparison between auditory and visual simple reaction times. Neurosci Med, 1: 30

[62] Ermolaeva VY, Borgest A (1980). Intercortical connections of the auditory areas with the motor area. Neurosci Behav Physiol, 10: 210-215

[63] Felix RA, Fridberger A, Leijon S, Berrebi AS, Magnusson AK (2011). Sound rhythms are encoded by postinhibitory rebound spiking in the superior paraolivary nucleus. J Neurosci, 31: 12566-12578

[64] Shannon K (2008). The effect of rhythmic auditory stimulation on the gait parameters of patients with incomplete spinal cord injury: an exploratory pilot study. Int J Rehabil Res, 31: 155-157

[65] Shahraki M, Sohrabi M, Torbati HT, Nikkhah K, NaeimiKia M (2017). Effect of rhythmic auditory stimulation on gait kinematic parameters of patients with multiple sclerosis. J Med Life, 10: 33

[66] Nascimento LR, de Oliveira CQ, Ada L, Michaelsen SM, Teixeira-Salmela LF (2015). Walking training with cueing of cadence improves walking speed and stride length after stroke more than walking training alone: a systematic review. J Physiother, 61: 10-15

[67] Lohnes CA, Earhart GM (2011). The impact of attentional, auditory, and combined cues on walking during single and cognitive dual tasks in Parkinson disease. Gait Posture, 33: 478-483

[68] Franěk M, van Noorden L, Režný L (2014). Tempo and walking speed with music in the urban context. Front Psychol, 5: 1361

[69] Deandrea S, Lucenteforte E, Bravi F, Foschi R, La Vecchia C, Negri E (2010). Risk Factors for Falls in Community-dwelling Older People:" A Systematic Review and Meta-analysis". Epidemiology: 658-668

[70] Thaut MH (2003). Neural basis of rhythmic timing networks in the human brain. Ann N Y Acad Sci, 999: 364-373

[71] Heremans E, Nieuwboer A, Feys P, Vercruysse S, Vandenberghe W, Sharma N, et al. (2012). External cueing improves motor imagery quality in patients with Parkinson disease. Neurorehabil Neural Repair, 26: 2735

[72] Heremans E, Nieuwboer A, Spildooren J, De Bondt S, D'hooge A-M, Helsen IW, et al. (2012). Cued motor imagery in patients with multiple sclerosis. Neuroscience, 206: 115-121

[73] Sigrist R, Rauter G, Riener R, Wolf P (2013). Augmented visual, auditory, haptic, and multimodal feedback in motor learning: a review. Psychon Bull Rev, 20: 21-53

[74] Keller PE, Dalla Bella S, Koch I (2010). Auditory imagery shapes movement timing and kinematics: Evidence from a musical task. J Exp Psychol Hum Percept, 36: 508

[75] Miller RA, Thaut MH, McIntosh GC, Rice RR (1996). Components of EMG symmetry and variability in 
parkinsonian and healthy elderly gait. Electroencephalogr Clin Neurophysiol, 101: 1-7

[76] Rochester L, Baker K, Nieuwboer A, Burn D (2011). Targeting dopa-sensitive and dopa-resistant gait dysfunction in Parkinson's disease: Selective responses to internal and external cues. Mov Disord, 26: 430-435

[77] Zhao Y, Nonnekes J, Storcken EJ, Janssen S, Wegen EE, Bloem BR, et al. (2016). Feasibility of external rhythmic cueing with the Google Glass for improving gait in people with Parkinson's disease. J Neurol, 263: 1156-1165

[78] Rodger MWM, Craig CM (2016). Beyond the Metronome: Auditory Events and Music May Afford More than Just Interval Durations as Gait Cues in Parkinson's Disease. Front Neurosci, 10: 272

[79] Espay AJ, Baram Y, Dwivedi AK, Shukla R, Gartner M, Gaines L, et al. (2010). At-home training with closed-loop augmented-reality cueing device for improving gait in patients with Parkinson disease. $\mathrm{J}$ Rehabil Res Dev, 47: 573

[80] Pau M, Corona F, Pili R, Casula C, Sors F, Agostini T, et al. (2016). effects of Physical rehabilitation integrated with rhythmic auditory stimulation on spatioTemporal and Kinematic Parameters of gait in Parkinson's Disease. Front Neurol, 7

[81] Peters DH, Garg A, Bloom G, Walker DG, Brieger WR, Hafizur Rahman M (2008). Poverty and access to health care in developing countries. Ann N Y Acad Sci, 1136: 161-171

[82] Liberati A, Altman DG, Tetzlaff J, Mulrow C, Gøtzsche PC, Ioannidis JP, et al. (2009). The PRISMA statement for reporting systematic reviews and meta-analyses of studies that evaluate health care interventions: explanation and elaboration. Ann Intern Med, 151: W65-W-94

[83] de Morton NA (2009). The PEDro scale is a valid measure of the methodological quality of clinical trials: a demographic study. Aust J Physiother, 55: 129-133

[84] Maher CG, Sherrington C, Herbert RD, Moseley AM, Elkins M (2003). Reliability of the PEDro scale for rating quality of randomized controlled trials. Phys Ther, 83: 713-721

[85] Teasell R, Foley N, Salter K, Bhogal S, Jutai J, Speechley M (2009). Evidence-Based Review of Stroke Rehabilitation: executive summary, 12th edition. Top Stroke Rehabil, 16: 463-488

[86] Ramsey L, Winder RJ, McVeigh JG (2014). The effectiveness of working wrist splints in adults with rheumatoid arthritis: A mixed methods systematic review. J Rehabil Med, 46: 481-492

[87] Borenstein M, Hedges LV, Higgins J, Rothstein HR (2010). A basic introduction to fixed-effect and random-effects models for meta-analysis. Res Synth Methods, 1: 97-111

[88] Higgins JP, Green S (2011) Cochrane handbook for systematic reviews of interventions Vol. 4, John Wiley \& Sons

[89] Cumming G (2013) Understanding the new statistics: Effect sizes, confidence intervals, and meta-analysis, Routledge
[90] Cohen J (1988) Statistical power analysis for the behavioral sciences, L, Erlbaum Associates, Hillsdale, $\mathrm{NJ}$

[91] Wellner M, Schaufelberger A, Zitzewitz Jv, Riener R (2008). Evaluation of visual and auditory feedback in virtual obstacle walking. Presence (Camb), 17: 512-524

[92] Almeida FAM, Nunes RFH, dos Santos Ferreira S, Krinski K, Elsangedy HM, Buzzachera CF, et al. (2015). Effects of musical tempo on physiological, affective, and perceptual variables and performance of self-selected walking pace. J Phys Ther Sci, 27: 17091712

[93] Yu L, Zhang Q, Hu C, Huang Q, Ye M, Li D (2015). Effects of different frequencies of rhythmic auditory cueing on the stride length, cadence, and gait speed in healthy young females. J Phys Ther Sci, 27: 485-487

[94] Rochester L, Hetherington V, Jones D, Nieuwboer A, Willems A-M, Kwakkel G, et al. (2005). The effect of external rhythmic cues (auditory and visual) on walking during a functional task in homes of people with Parkinson's disease. Arch Phys Med Rehabil, 86: 9991006

[95] Baram Y, Aharon-Peretz J, Badarny S, Susel Z, Schlesinger I (2016). Closed-loop auditory feedback for the improvement of gait in patients with Parkinson's disease. J Neurol Sci, 363: 104-106

[96] Terrier P (2016). Fractal fluctuations in human walking: comparison between auditory and visually guided stepping. Ann Biomed Eng, 44: 2785-2793

[97] Schreiber C, Remacle A, Chantraine F, Kolanowski E, Moissenet F (2016). Influence of a rhythmic auditory stimulation on asymptomatic gait. Gait Posture, 50: 1722

[98] Bolier L, Haverman M, Westerhof GJ, Riper H, Smit F, Bohlmeijer E (2013). Positive psychology interventions: a meta-analysis of randomized controlled studies. BMC public health, 13: 119

[99] Baram Y, Miller A (2007). Auditory feedback control for improvement of gait in patients with Multiple Sclerosis. J Neurol Sci, 254: 90-94

[100] Dotov D, Bayard S, de Cock VC, Geny C, Driss V, Garrigue G, et al. (2017). Biologically-variable rhythmic auditory cues are superior to isochronous cues in fostering natural gait variability in Parkinson's disease. Gait Posture, 51: 64-69

[101] Baker K, Rochester L, Nieuwboer A (2007). The immediate effect of attentional, auditory, and a combined cue strategy on gait during single and dual tasks in Parkinson's disease. Arch Phys Med Rehabil, 88: $1593-1600$

[102] Arias P, Cudeiro J (2008). Effects of rhythmic sensory stimulation (auditory, visual) on gait in Parkinson's disease patients. Exp Brain Res, 186: 589-601

[103] Willems AM, Nieuwboer A, Chavret F, Desloovere K, Dom R, Rochester L, et al. (2007). Turning in Parkinson's disease patients and controls: the effect of auditory cues. Mov Disord, 22: 1871-1878

[104] Hamacher D, Hamacher D, Herold F, Schega L (2016). Effect of dual tasks on gait variability in walking to 
auditory cues in older and young individuals. Exp Brain Res, 234: 3555-3563

[105] Leow L-A, Parrott T, Grahn JA (2014). Individual differences in beat perception affect gait responses to low-and high-groove music. Front Hum Neurosci, 8: 811

[106] Hollman JH, McDade EM, Petersen RC (2011). Normative Spatiotemporal Gait Parameters in Older Adults. Gait Posture, 34: 111-118

[107] Callisaya ML, Beare R, Phan TG, Blizzard L, Thrift AG, Chen J, et al. (2013). Brain structural change and gait decline: a longitudinal population-based study. J Am Geriatr Soc, 61: 1074-1079

[108] Aboutorabi A, Arazpour M, Bahramizadeh M, Hutchins SW, Fadayevatan R (2016). The effect of aging on gait parameters in able-bodied older subjects: a literature review. Aging Clin Exp Res, 28: 393-405

[109] Raz N, Rodrigue KM, Kennedy KM, Head D, GunningDixon F, Acker JD (2003). Differential aging of the human striatum: longitudinal evidence. Am J Neuroradiol, 24: 1849-1856

[110] Wolpe N, Ingram JN, Tsvetanov KA, Geerligs L, Kievit RA, Henson RN, et al. (2016). Ageing increases reliance on sensorimotor prediction through structural and functional differences in frontostriatal circuits. Nat Commun, 7

[111] Seidler RD, Bernard JA, Burutolu TB, Fling BW, Gordon MT, Gwin JT, et al. (2010). Motor Control and Aging: Links to Age-Related Brain Structural, Functional, and Biochemical Effects. Neurosci Biobehav Rev, 34: 721-733

[112] Perry MC, Carville SF, Smith ICH, Rutherford OM, Newham DJ (2007). Strength, power output and symmetry of leg muscles: effect of age and history of falling. Eur J Appl Physiol, 100: 553-561

[113] Rizzo J-R, Raghavan P, McCrery JR, Oh-Park M, Verghese J (2015). Effects of Emotionally Charged Auditory Stimulation on Gait Performance in the Elderly: A Preliminary Study. Arch Phys Med Rehabil, 96: 690-696

[114] Schmidt RA (1991) Frequent augmented feedback can degrade learning: Evidence and interpretations. In Tutorials in motor neuroscience pp. 59-75, Springer

[115] Winstein CJ, Pohl PS, Lewthwaite R (1994). Effects of physical guidance and knowledge of results on motor learning: support for the guidance hypothesis. Res Q Exerc Sport, 65: 316-323

[116] Elsinger CL, Rao SM, Zimbelman JL, Reynolds NC, Blindauer KA, Hoffmann RG (2003). Neural basis for impaired time reproduction in Parkinson's disease: an fMRI study. J Int Neuropsychol Soc, 9: 1088-1098

[117] Hausdorff JM, Lowenthal J, Herman T, Gruendlinger L, Peretz C, Giladi N (2007). Rhythmic auditory stimulation modulates gait variability in Parkinson's disease. Eur J Neurosci, 26: 2369-2375

[118] Rubinstein TC, Giladi N, Hausdorff JM (2002). The power of cueing to circumvent dopamine deficits: a review of physical therapy treatment of gait disturbances in Parkinson's disease. Mov Disord, 17: 1148-1160
[119] Cunnington R, Iansek R, Bradshaw JL, Phillips JG (1995). Movement-related potentials in Parkinson's disease. Brain, 118: 935-950

[120] Fujioka T, Trainor LJ, Large EW, Ross B (2012). Internalized timing of isochronous sounds is represented in neuromagnetic beta oscillations. $\mathbf{J}$ Neurosci, 32: 1791-1802

[121] Cabeza R, Anderson ND, Locantore JK, McIntosh AR (2002). Aging gracefully: compensatory brain activity in high-performing older adults. Neuroimage, 17: 13941402

[122] Tierney A, Kraus N (2013). The ability to move to a beat is linked to the consistency of neural responses to sound. J Neurosci, 33: 14981-14988

[123] Debaere F, Wenderoth N, Sunaert S, Van Hecke P, Swinnen SP (2003). Internal vs external generation of movements: differential neural pathways involved in bimanual coordination performed in the presence or absence of augmented visual feedback. Neuroimage, 19: 764-776

[124] Asanuma H, Keller A (1991). Neuronal mechanisms of motor learning in mammals. Neuroreport, 2: 217-224

[125] Suh JH, Han SJ, Jeon SY, Kim HJ, Lee JE, Yoon TS, et al. (2014). Effect of rhythmic auditory stimulation on gait and balance in hemiplegic stroke patients. NeuroRehabilitation, 34: 193-199

[126] Luft AR, McCombe-Waller S, Whitall J, Forrester LW, Macko R, Sorkin JD, et al. (2004). Repetitive bilateral arm training and motor cortex activation in chronic stroke: a randomized controlled trial. Jama, 292: 18531861

[127] Thaut MH, Gardiner JC, Holmberg D, Horwitz J, Kent L, Andrews G, et al. (2009). Neurologic music therapy improves executive function and emotional adjustment in traumatic brain injury rehabilitation. Ann N Y Acad Sci, 1169: 406-416

[128] Delignières D, Torre K (2009). Fractal dynamics of human gait: a reassessment of the 1996 data of Hausdorff et al. J Appl Physiol, 106: 1272-1279

[129] Hove MJ, Suzuki K, Uchitomi H, Orimo S, Miyake Y (2012). Interactive rhythmic auditory stimulation reinstates natural 1/f timing in gait of Parkinson's patients. PloS one, 7: e32600

[130] Hausdorff JM, Purdon PL, Peng C, Ladin Z, Wei JY, Goldberger AL (1996). Fractal dynamics of human gait: stability of long-range correlations in stride interval fluctuations. J Appl Physiol, 80: 1448-1457

[131] Snijders A, Verstappen C, Munneke M, Bloem B (2007). Assessing the interplay between cognition and gait in the clinical setting. J Neural Transm, 114: 13151321

[132] Muir-Hunter SW, Wittwer JE (2016). Dual-task testing to predict falls in community-dwelling older adults: a systematic review. Physiotherapy, 102: 29-40

[133] Bock O (2008). Dual-task costs while walking increase in old age for some, but not for other tasks: an experimental study of healthy young and elderly persons. J Neuroeng Rehabil, 5: 27-27

[134] O'Shea S, Morris ME, Iansek R (2002). Dual task interference during gait in people with Parkinson 
disease: effects of motor versus cognitive secondary tasks. Phys Ther, 82: 888-897

[135] Morris ME, Iansek R, Matyas TA, Summers JJ (1996). Stride length regulation in Parkinson's disease: normalization strategies and underlying mechanisms. Brain, 119: 551-568

[136] Wittwer JE, Webster KE, Hill K (2013). Music and metronome cues produce different effects on gait spatiotemporal measures but not gait variability in healthy older adults. Gait Posture, 37: 219-222

[137] Thaut MH, Miltner R, Lange HW, Hurt CP, Hoemberg $\mathrm{V}$ (1999). Velocity modulation and rhythmic synchronization of gait in Huntington's disease. Mov Disord, 14: 808-819

[138] Young WR, Rodger MW, Craig CM (2014). Auditory observation of stepping actions can cue both spatial and temporal components of gait in Parkinson' s disease patients. Neuropsychologia, 57: 140-153

[139] Gaver WW (1993). How do we hear in the world? Explorations in ecological acoustics. Ecol Psychol, 5: 285-313

[140] Young W, Rodger M, Craig CM (2013). Perceiving and reenacting spatiotemporal characteristics of walking sounds. J Exp Psychol Hum Percept, 39: 464

[141] Maculewicz J, Erkut C, Serafin S (2016). An investigation on the impact of auditory and haptic feedback on rhythmic walking interactions. Int J Hum Comput Stud, 85: 40-46

[142] Leman M, Moelants D, Varewyck M, Styns F, van Noorden L, Martens J-P (2013). Activating and relaxing music entrains the speed of beat synchronized walking. PloS one, 8: e67932

[143] Horst F, Eekhoff A, Newell KM, Schöllhorn WI (2017). Intra-individual gait patterns across different timescales as revealed by means of a supervised learning model using kernel-based discriminant regression. PLoS ONE, 12: e0179738

[144] Clark JE, Phillips SJ (1993). A longitudinal study of intralimb coordination in the first year of independent walking: a dynamical systems analysis. Child Dev, 64: 1143-1157

[145] Stergiou N, Decker LM (2011). Human movement variability, nonlinear dynamics, and pathology: is there a connection? Hum Mov Sci, 30: 869-888

[146] del Olmo MF, Arias P, Furio M, Pozo M, Cudeiro J (2006). Evaluation of the effect of training using auditory stimulation on rhythmic movement in Parkinsonian patients - a combined motor and [18 F]FDG PET study. Parkinsonism Relat Disord, 12: 155164

[147] Fang R, Ye S, Huangfu J, Calimag DP (2017). Music therapy is a potential intervention for cognition of Alzheimer's Disease: a mini-review. Transl Neurodegener, 6: 2

[148] Hanna-Pladdy B, MacKay A (2011). The Relation Between Instrumental Musical Activity and Cognitive Aging. Neuropsychology, 25: 378-386

[149] Sturman MT, Morris MC, Mendes de Leon CF, Bienias JL, Wilson RS, Evans DA (2005). Physical activity, cognitive activity, and cognitive decline in a biracial community population. Arch Neurol, 62: 1750-1754

[150] Mammarella N, Fairfield B, Cornoldi C (2007). Does music enhance cognitive performance in healthy older adults? The Vivaldi effect. Aging Clin Exp Res, 19: 394-399

[151] Stork MJ, Kwan MY, Gibala MJ, Martin Ginis KA (2015). Music enhances performance and perceived enjoyment of sprint interval exercise. Med Sci Sports Exerc, 47: 1052-1060

[152] Menon V, Levitin DJ (2005). The rewards of music listening: response and physiological connectivity of the mesolimbic system. Neuroimage, 28: 175-184

[153] Eliakim M, Meckel Y, Nemet D, Eliakim A (2007). The effect of music during warm-up on consecutive anaerobic performance in elite adolescent volleyball players. Int J Sports Med, 28: 321-325

[154] Crust L (2004). Carry-Over Effects of Music in an Isometric Muscular Endurance Task. Perceptual and Motor Skills, 98: 985-991

[155] Waxman A (2005) Why a global strategy on diet, physical activity and health? In Nutrition and Fitness: Mental Health, Aging, and the Implementation of a Healthy Diet and Physical Activity Lifestyle Vol. 95 pp. 162-166, Karger Publishers

[156] Rochester L, Rafferty D, Dotchin C, Msuya O, Minde V, Walker R (2010). The effect of cueing therapy on single and dual-task gait in a drug naïve population of people with Parkinson's disease in northern Tanzania. Mov Disord, 25: 906-911

[157] Godara B, Nikita KS (2013) Wireless Mobile Communication and Healthcare: Third International Conference, MobiHealth 2012, Paris, France, November 21-23, 2012, Revised Selected Papers Vol. 61, Springer

[158] Lopez WO, Higuera CA, Fonoff ET, Souza Cde O, Albicker U, Martinez JA (2014). Listenmee and Listenmee smartphone application: synchronizing walking to rhythmic auditory cues to improve gait in Parkinson's disease. Hum Mov Sci, 37: 147-156

[159] Poushter J (2016). Smartphone ownership and internet usage continues to climb in emerging economies. 2016. URL: http://www. pewglobal. org, 2: 22

[160] Ghai S, Ghai I, Effenberg AO (2017). Effect of rhythmic auditory cueing on gait in Cerebral palsy: A systematic review and meta-analysis. Neuropsychiatr Dis Treat, Accepted, In Press

[161] Rocha PA, Porfírio GM, Ferraz HB, Trevisani VF (2014). Effects of external cues on gait parameters of Parkinson's disease patients: a systematic review. Clin Neurol Neurosurg, 124: 127-134

[162] Roerdink M, Daffertshofer A, Marmelat V, Beek PJ (2015). How to sync to the beat of a persistent fractal metronome without falling off the treadmill? PloS one, 10: e0134148

[163] Wright RL, Spurgeon LC, Elliott MT (2014). Stepping to phase-perturbed metronome cues: multisensory advantage in movement synchrony but not correction. Front Hum Neurosci, 8: 724 
[164] Sejdić E, Fu Y, Pak A, Fairley JA, Chau T (2012). The effects of rhythmic sensory cues on the temporal dynamics of human gait. PloS one, 7: e43104

[165] Terrier P, Dériaz O (2012). Nonlinear dynamics of human locomotion: effects of rhythmic auditory cueing on local dynamic stability. arXiv preprint arXiv:1211.3616,

[166] Roerdink M, Bank PJ, Peper CLE, Beek PJ (2011). Walking to the beat of different drums: Practical implications for the use of acoustic rhythms in gait rehabilitation. Gait Posture, 33: 690-694

[167] Trombetti A, Hars M, Herrmann FR, Kressig RW, Ferrari S, Rizzoli R (2011). Effect of music-based multitask training on gait, balance, and fall risk in elderly people: a randomized controlled trial. Arch Intern Med, 171: 525-533

[168] Hunt N, McGrath D, Stergiou N (2014). The influence of auditory-motor coupling on fractal dynamics in human gait. Sci rep, 4: 5879

[169] Marmelat V, Torre K, Beek PJ, Daffertshofer A (2014). Persistent fluctuations in stride intervals under fractal auditory stimulation. PLoS One, 9: e91949

[170] Peper CLE, Oorthuizen JK, Roerdink M (2012). Attentional demands of cued walking in healthy young and elderly adults. Gait Posture, 36: 378-382
[171] Bank PJ, Roerdink M, Peper C (2011). Comparing the efficacy of metronome beeps and stepping stones to adjust gait: steps to follow! Exp Brain Res, 209: 159169

[172] Baker K, Rochester L, Nieuwboer A (2008). The effect of cues on gait variability-Reducing the attentional cost of walking in people with Parkinson's disease. Parkinsonism Relat Disord, 14: 314-320

[173] Willems A-M, Nieuwboer A, Chavret F, Desloovere K, Dom R, Rochester L, et al. (2006). The use of rhythmic auditory cues to influence gait in patients with Parkinson's disease, the differential effect for freezers and non-freezers, an explorative study. Disabil Rehabil, 28: 721-728

[174] Thaut MH, McIntosh GC, Prassas SG, Rice RR (1992). Effect of rhythmic auditory cuing on temporal stride parameters and EMG patterns in normal gait. J Neurol Rehabil, 6: 185-190

[175] McIntosh GC, Brown SH, Rice RR, Thaut MH (1997). Rhythmic auditory-motor facilitation of gait patterns in patients with Parkinson's disease. J Neurol Neurosurg Psychiatry, 62: 22-26 